\title{
Microsurgical anatomy of safe entry zones to the brainstem
}

\author{
Daniel D. Cavalcanti, MD, ${ }^{1}$ Mark C. Preul, MD, ${ }^{2}$ M. Yashar S. Kalani, MD, PhD, ${ }^{2}$ and \\ Robert F. Spetzler, MD²
}

\begin{abstract}
1Department of Neurosurgery, Paulo Niemeyer State Brain Institute, Rio de Janeiro, Brazil; and 2Division of Neurological Surgery, Barrow Neurological Institute, St. Joseph's Hospital and Medical Center, Phoenix, Arizona
\end{abstract}

OBJECTIVE The aim of this study was to enhance the planning and use of microsurgical resection techniques for intrinsic brainstem lesions by better defining anatomical safe entry zones.

METHODS Five cadaveric heads were dissected using 10 surgical approaches per head. Stepwise dissections focused on the actual areas of brainstem surface that were exposed through each approach and an analysis of the structures found, as well as which safe entry zones were accessible via each of the 10 surgical windows.

RESULTS Thirteen safe entry zones have been reported and validated for approaching lesions in the brainstem, including the anterior mesencephalic zone, lateral mesencephalic sulcus, intercollicular region, peritrigeminal zone, supratrigeminal zone, lateral pontine zone, supracollicular zone, infracollicular zone, median sulcus of the fourth ventricle, anterolateral and posterior median sulci of the medulla, olivary zone, and lateral medullary zone. A discussion of the approaches, anatomy, and limitations of these entry zones is included.

CONCLUSIONS A detailed understanding of the anatomy, area of exposure, and safe entry zones for each major approach allows for improved surgical planning and dissemination of the techniques required to successfully resect intrinsic brainstem lesions.

http://thejns.org/doi/abs/10.3171/2015.4.JNS141945

KEY WORDS brainstem; cavernous malformation; microsurgery; safe entry zones; surgical approaches; surgical anatomy

$\mathrm{H}$ ISTORICALLY, the surgical management of intrinsic brainstem lesions has been controversial. The surgical extirpation of focal gliomas, cavernous malformations, or hemangioblastomas within the brainstem has caused heated discussions in scientific meetings and the literature. In 1939, Bailey et al. ${ }^{3}$ declared this subject to be a pessimistic chapter in neurosurgery; 30 years later, Matson and Ingraham ${ }^{26}$ would still claim such lesions were inoperable. However, in 1971, Lassiter et al. ${ }^{25}$ were among the first to advocate surgical intervention. By 1986, Epstein and McCleary reported that surgery was feasible with reasonable morbidity and mortality. ${ }^{15}$ Concurrent with Epstein and McCleary's report, Raimondi would rationally state that to have the child merely survive (i.e., with severe neurological deficits) is no justification for surgery. ${ }^{33}$ The development and improvement of complex skull base surgical approaches and incremental advances in neuroimaging, parallel to image-guided surgery, allowed a few authors to safely and effectively resect lesions in the brainstem. $., 23,32$

Knowledge of different skull base exposures, gained through laboratory dissections, allows neurosurgeons to approach lesions in the brainstem. Nevertheless, the brainstem, roughly the size of the human thumb, contains a rich concentration of nuclei and fibers in a small sectional area, resulting in a high likelihood of morbidity after manipulation. Awareness of the main safe entry zones on the brainstem is key to reducing morbidity for any lesion that does not emerge to the pial or ependymal surface. Such zones represent entry points and trajectories where eloquent structures and perforators are sparse and where a neurotomy would cause the least possible damage.

ABBREVIATIONS CN = cranial nerve; mini-OZ = mini-modified orbitozygomatic approach; $\mathrm{PCA}=$ posterior cerebral artery; $\mathrm{P}_{2} \mathrm{P}=$ posterior $\mathrm{P}_{2} ; \mathrm{SCA}=$ superior cerebellar artery; TAPS = transanterior perforating substance.

SUBMITTED August 21, 2014. ACCEPTED April 8, 2015.

INCLUDE WHEN CITING Published online October 9, 2015; DOI: 10.3171/2015.4.JNS141945. 
The aim of our study was to enhance the planning and use of microsurgical resection techniques for intrinsic brainstem lesions. We examined 13 safe entry zones on the brainstem, which have been described in the literature, and used detailed cadaveric dissections to evaluate the main surgical approaches currently employed to manage intrinsic brainstem lesions. Through detailed dissection images of these approaches, we visually demonstrate what can be seen on the brainstem surface through each of these corridors and delineate the safe entry zones provided by each approach. It is critical to note that large lesions may distort safe entry zones and that neurophysiological monitoring is a critical adjunct in these cases. ${ }^{36}$

\section{Methods}

We searched MEDLINE and Google Scholar for studies containing terms related to the surgical management of brainstem lesions to determine the most frequently discussed safe entry zones. Thirteen zones were selected: 1) anterior mesencephalic zone, 2) lateral mesencephalic sulcus, 3) intercollicular region, 4) peritrigeminal zone, 5) supratrigeminal zone, 6) lateral pontine zone, 7) supracollicular zone, 8) infracollicular zone, 9) median sulcus of the fourth ventricle, 10) anterolateral and 11) posterior median sulci of the medulla, 12) olivary zone, and 13) lateral medullary zone. . $^{5,9,14,20,23,35}$

Five human cadaveric heads, formalin-fixed and injected with colored silicone rubber, were carefully dissected in a simulated surgical environment at the Skull Base Laboratory of the Barrow Neurological Institute in Phoenix, Arizona. With the heads fixed with Mayfield clamps, 10 surgical approaches were performed on each head: 1) orbitozygomatic, 2) subtemporal, 3) subtemporal transtentorial, 4) anterior petrosectomy, 5) suboccipital telovelar, 6) median supracerebellar infratentorial, 7) extreme lateral supracerebellar infratentorial, 8) retrosigmoid, 9) far lateral, and 10) retrolabyrinthine. The order in which the approaches were performed was altered for each head to obtain clear images of each approach. Every step was photographically registered, and the resultant surgical exposure on the brainstem surface for each approach was analyzed for both identifiable anatomical structures and the safe entry zones that were available.

\section{Results}

\section{Safe Entry Zones}

The safe entry zones discussed can be divided into 3 main regions: midbrain (Fig. 1), pons (Fig. 2), and medulla oblongata (Fig. 3).

\section{Midbrain}

\section{Anterior Mesencephalic Zone}

Lesions involving the anterior midbrain can be accessed through a limited area on the cerebral peduncle bounded medially by the oculomotor tract and nerve and laterally by the corticospinal tract (Fig. 1A and B). Such a narrow corridor takes advantage of the distribution of corticospinal tract fibers mainly in the intermediate threefifths of the peduncle and the fact that the red nucleus and the nigrostriatal circuit are in a deep medial location. ${ }^{5}$ The entry point inside the interpeduncular cistern is limited superiorly by the posterior cerebral artery (PCA) and inferiorly by the main trunk of the superior cerebellar artery (SCA) (Figs. 4F and $\mathrm{H}$ and 5F).

\section{Lateral Mesencephalic Sulcus}

Hidden by the lateral mesencephalic vein, the lateral mesencephalic sulcus separates the peduncular and tegmental surfaces of the midbrain facing the middle incisural space. ${ }^{30}$ The lateral mesencephalic sulcus extends downward in a concave fashion from the medial geniculate body to the pontomesencephalic sulcus (Fig. $1 \mathrm{~A}$ and $\mathrm{C}$ ). Crossing the sulcus are the posterior $\mathrm{P}_{2}$ segment $\left(\mathrm{P}_{2} \mathrm{P}\right)$ superiorly, the medial posterior choroidal artery centrally, and the cerebellomesencephalic segments of the SCA, trochlear nerve, and tentorial edge inferiorly (Fig. 5G and H).

The safe entry zone runs between the substantia nigra anterolaterally and the medial lemniscus posteriorly. The oculomotor nerve fibers crossing from the red nucleus to the substantia nigra impose an anteromedial limit to dissection. Recalde et al. ${ }^{35}$ found that the average total length of the sulcus was $9.6 \mathrm{~mm}$ (range 7.4-13.3 mm) with an average working-channel length of $8.0 \mathrm{~mm}$ (range 4.9-11.7 $\mathrm{mm})$.

\section{Intercollicular Region}

The quadrigeminal plate or tectum is composed of 2 superior and 2 inferior rounded eminences, designated as superior colliculi and inferior colliculi, respectively, which represent the dorsal surface of the midbrain (Fig. $1 \mathrm{~A}$ and $\mathrm{D})$.

The superior colliculi are active in the visual system; they are critical to visual fixation and saccadic eye movements. ${ }^{19,28}$ They are connected to each lateral geniculate body by a superior brachium, a path in which the retinotectal fibers run. ${ }^{21}$ Additionally, spinotectal and corticotectal fibers reach the superior colliculi, while tectospinal, tectothalamic, and tectocortical tracts leave these structures.

The inferior colliculi are part of the auditory system. They receive fibers from the contralateral cochlear nucleus, dorsal and ventral nuclei of the lateral lemniscus, contralateral and ipsilateral superior olive, ipsilateral medial superior olive, and descending projections from sensory areas through the corticollicular neurons ${ }^{8,27}$ Commissural fibers connect 1 inferior colliculi to another. ${ }^{27}$ The inferior colliculi extends laterally through the inferior brachium to the medial geniculate body of the thalamus, which projects to the primary auditory cortex.

The most appropriate area for a small neurotomy has been described as the intercollicular region, because of its sparseness of fibers (Fig. 1A and D). Bricolo and Turazzi ${ }^{5}$ first suggested this corridor, which has been further supported by other studies. ${ }^{9,22,34}$

\section{Pons}

\section{Peritrigeminal Zone}

The anterolateral surface of the pons has traditionally been considered a safe zone for entering the brainstem., ${ }^{9,17,32}$ Using the white fiber dissection technique, Recalde et al ${ }^{35}$ were able to clearly quantify a safe trajectory 

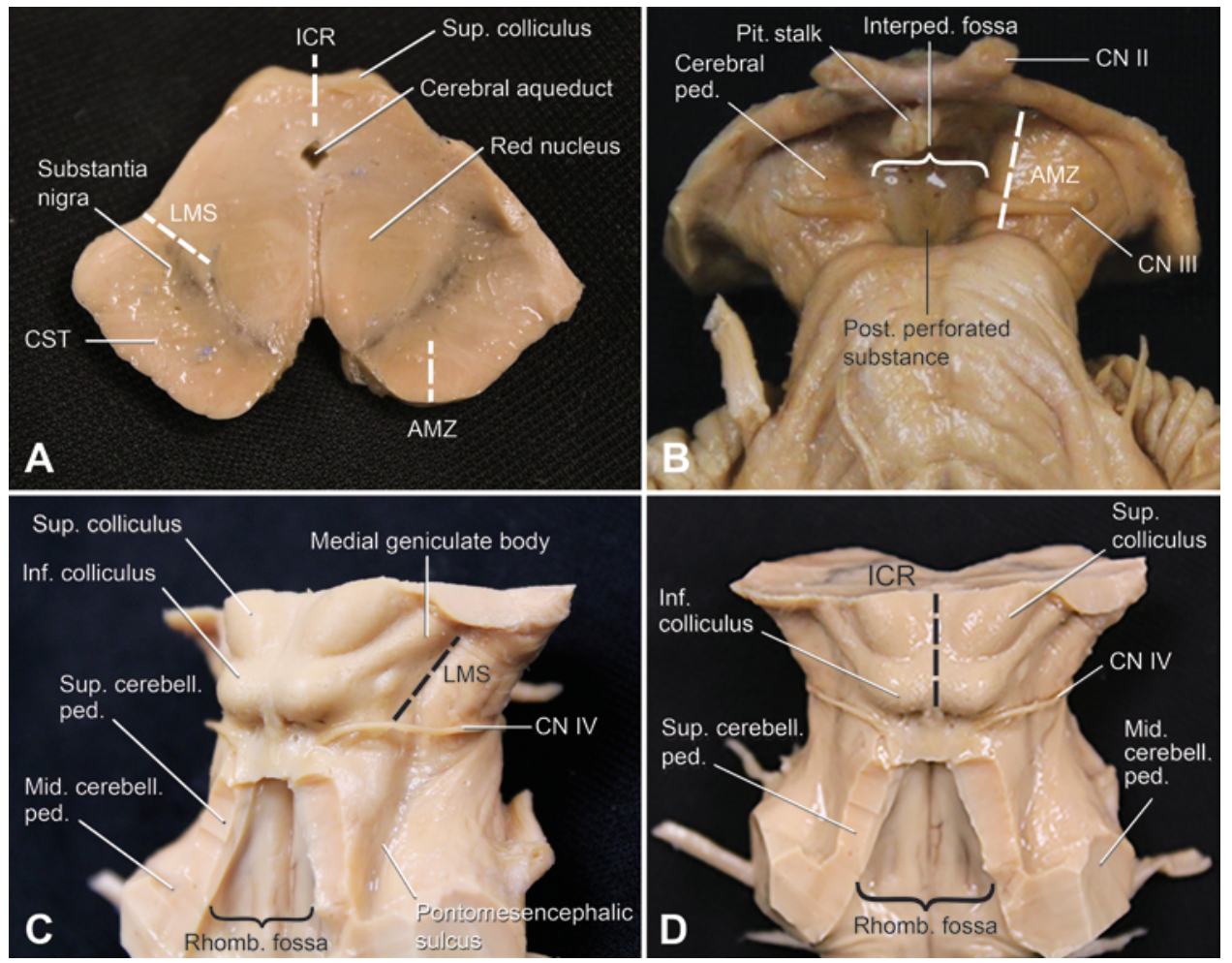

FIG. 1. Safe entry zones of the midbrain. A: Cross section of the midbrain at the level of the cerebral peduncle revealing its 3 safe entry zones (white dashed lines): the lateral mesencephalic sulcus (LMS), the intercollicular region (ICR), and the anterior mesencephalic zone (AMZ). B: Anterior view of a dissected brainstem revealing the AMZ (white dashed line). C: Right posterolateral view of the brainstem showing the LMS (black dashed line). D: Posterior exposure of the brainstem showing the ICR (black dashed line). Cerebral ped. = cerebral peduncle; CST = corticospinal tract; inf. = inferior; interped. fossa = interpeduncular fossa; mid. cerebell. ped. $=$ middle cerebellar peduncle; pit. stalk = pituitary stalk; post. = posterior; rhomb. fossa = rhomboid fossa; sup = superior; sup. cerebell. ped. $=$ superior cerebellar peduncle.
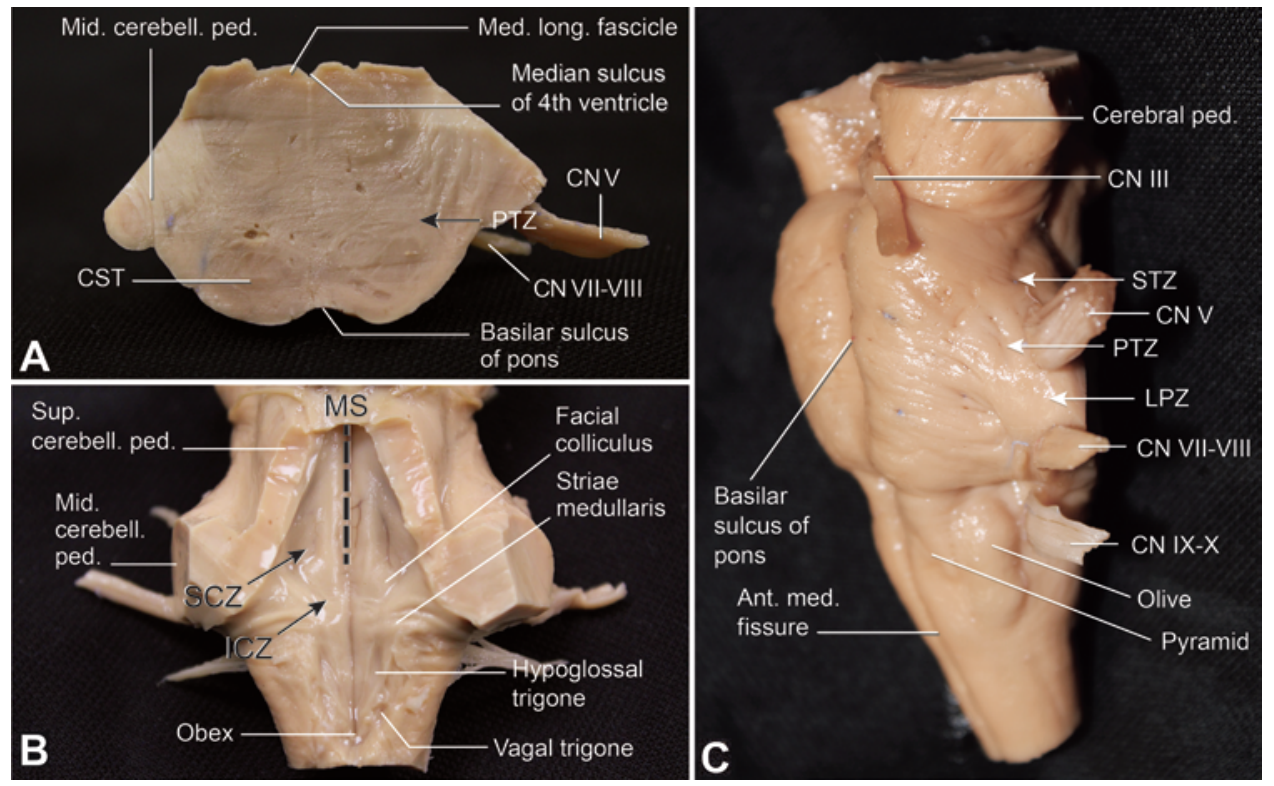

FIG. 2. Safe entry zones of the pons. A: Cross section of the pons demonstrating the peritrigeminal zone (PTZ; arrow). B: Three entry zones can be used on the rhomboid fossa: the supracollicular zone (SCZ; arrow), the infracollicular zone (ICZ; arrow), and the median sulcus of the fourth ventricle (MS; dashed line). C: The arrows represent the safe entry zones for excising lateral and anterolateral pontine lesions: the supratrigeminal zone (STZ), the PTZ, and the lateral pontine zone (LPZ). Ant. med. fissure = anterior median fissure; med. long. fascicle = medial longitudinal fascicle. 

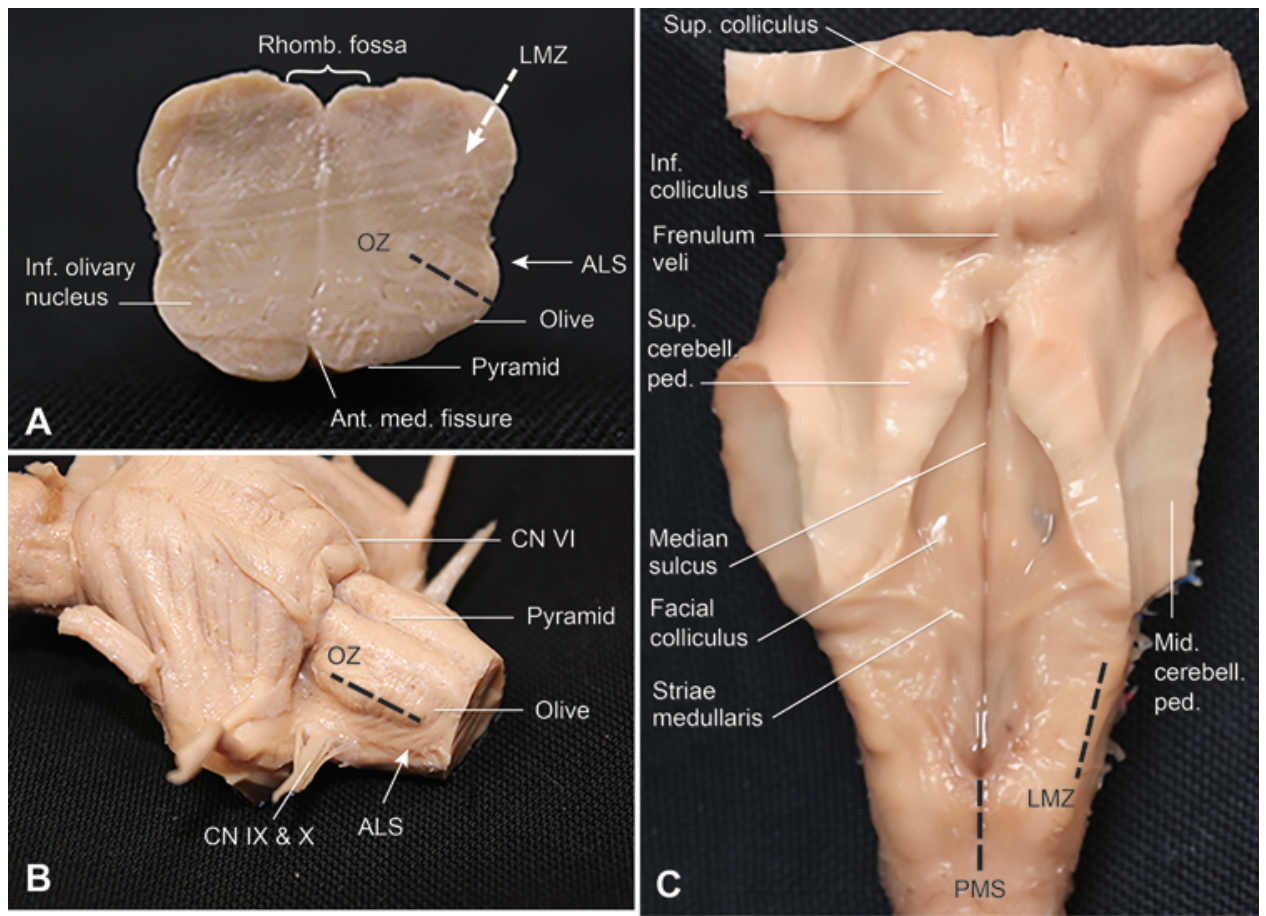

FIG. 3. Safe entry zones of the medulla oblongata. A: Cross section of the medulla revealing the 3 safe entry zones: the anterolateral sulcus (ALS; arrow), the olivary zone (OZ; dashed line), and the posterolateral lateral medullary zone (LMZ; dashed arrow). B: Anterolateral view of a dissected brainstem showing the site for entering the olive (dashed line) and the entry site (arrow) on the ALS just below the root of the hypoglossal nerve. C: Posterior view of the brainstem revealing the posterior median sulcus (PMS) and LMZ safe zones. Ant. med. fissure = anterior median fissure.

in front of the trigeminal nerve (cranial nerve $\mathrm{V}[\mathrm{CN} \mathrm{V}]$ ) entry zone, lateral to the corticospinal tract and anterior to the motor and sensory nuclei of the trigeminal nerve (Fig. 2A and B). On the axial plane, they found a mean distance of $4.64 \mathrm{~mm}$ (range 3.8-5.6 $\mathrm{mm}$ ) between $\mathrm{CN} \mathrm{V}$ and the corticospinal tract, and a mean depth of dissection of $11.2 \mathrm{~mm}$ (range $9.5-13.1 \mathrm{~mm}$ ) to the trigeminal nuclei. The fibers of CNs VI, VII, and VIII run downward and are located posterior to the trigeminal nuclei.

\section{Supratrigeminal Zone}

A second entry point that has been used to manage anteriorly placed lesions in the pons is located just above the trigeminal root entry zone on the middle cerebellar peduncle (Fig. 2B) ${ }^{20}$ Taking advantage of the posterolateral location of the middle cerebellar peduncle and the thick pontine transverse fibers, it is possible to carefully dissect along these fibers, medially or anteromedially, posterior to the trajectory of the corticospinal tract.

\section{Lateral Pontine Zone}

In 1982, Baghai et al. ${ }^{2}$ recommended a safe corridor on the junction between the middle cerebellar peduncle and the pons and between the trigeminal and the facial-vestibulocochlear complex root entry zones (Fig. 2B). Other authors have supported using the narrow corridor of the lateral pontine zone, but it restrains vertical manipulation. ${ }^{5}$

\section{Supracollicular and Infracollicular Zones}

The rhomboid fossa hides several structures whose manipulation may result in increased morbidity. Superficial landmarks help protect crucial structures located at the depth of the fourth ventricular floor. At the floor of the fourth ventricle, the facial nerve passes around the nucleus of the abducens nerve; this combined structure is called the facial colliculus. The medial longitudinal fascicle lies parallel to the median sulcus. Similarly, the nuclei of CNs $\mathrm{X}$ and XII are located just caudal to the striae medullaris.

After examining the topographic anatomy of the facial colliculi and every potential sign of neurological deficit presented after manipulating the rhomboid fossa, Kyoshima et al. ${ }^{23}$ described 2 safe zones through this region that minimally displace surrounding neural structures (Fig. 2C, with specific reference to SCZ and ICZ). The first safe zone is the suprafacial triangle, which is delimited caudally by the facial nerve, laterally by the cerebellar peduncles, and medially by the medial longitudinal fascicle. The second safe zone is described by the edges of the infrafacial triangle, which are the striae medullaris caudally, the facial nerve laterally, and the medial longitudinal fascicle medially.

However, Strauss et al. ${ }^{37}$ stressed the variability of the striae medullaris and the possible damage to either the trigeminal motor nucleus or the nuclei of the lower cranial nerves when using the suprafacial or infrafacial triangles, respectively. They conducted a detailed morphometric study to measure the dimensions of the facial colliculus and its distance to the midline, decussation of CN IV, and vagal and hypoglossal trigones. They defined a paramedian supracollicular area that measured $13.8 \mathrm{~mm}$ vertically between the facial colliculus and the decussation of 

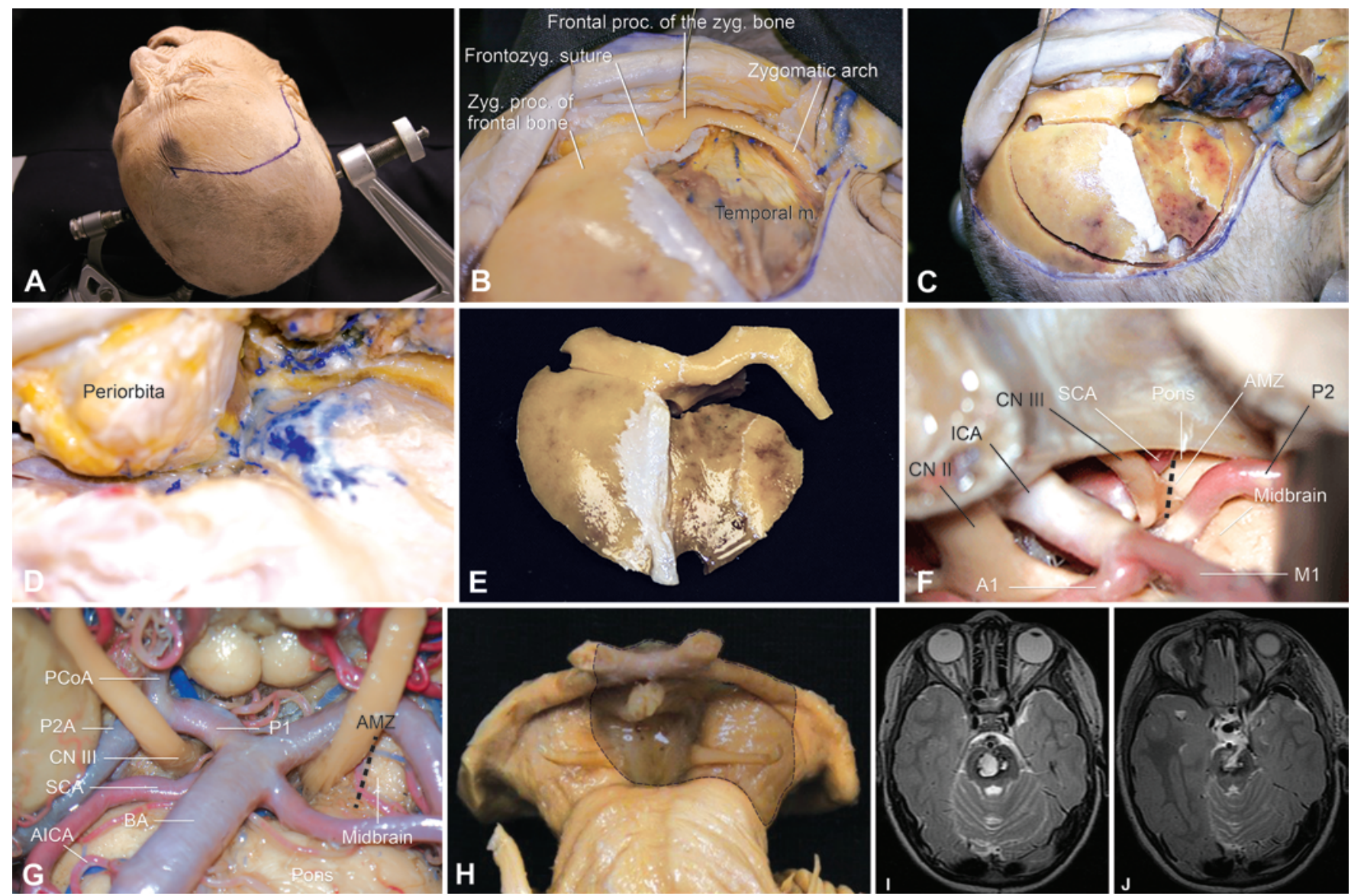

FIG. 4. Orbitozygomatic approach. A: A hemicoronal incision begins in the preauricular area and curves forward at the midline. B: The skin flap is reflected along with the temporal fascia. A subperiosteal dissection reveals the zygomatic process of the frontal bone, the frontal process of the zygomatic bone, and the zygomatic arch. C: A pterional craniotomy is prepared after detaching the temporal muscle. D: Magnified view after elevating the pterional flap and completing the orbitozygomatic osteotomy. E: The 2-piece craniotomy flap. F: The surgical window provided by this approach, centered on the exit of the oculomotor nerve from the cerebral peduncle. The anterior mesencephalic zone (AMZ) is shown by the dashed line. G: Cadaveric dissection demonstrating the AMZ, limited superiorly by the posterior cerebral artery, inferiorly by the superior cerebellar artery, medially by the oculomotor nerve, and laterally by the projection of the corticospinal tract fibers on the surface. H: Shaded area represents the area of exposure provided by the orbitozygomatic approach. I: Preoperative T2-weighted MR image in a 3-year-old male reveals the characteristic imaging findings of a cavernous malformation. The lesion was approached via a left-modified orbitozygomatic approach via the anterior mesencephalic sulcus. J: Postoperative T2-weighted MR image reveals gross-total resection of the lesion. $\mathrm{AICA}=$ anterior inferior cerebellar artery; $\mathrm{BA}=$ basilar artery; frontozyg. = frontozygomatic; ICA = internal carotid artery; $\mathrm{m} .=$ muscle; $M 1=M_{1}$ segment of the middle cerebral artery; $P 2 A=$ anterior part of the $P_{2}$ segment of the posterior cerebral artery; $\mathrm{PCA}(\mathrm{P} 1)=$ posterior cerebral artery $\left(\mathrm{P}_{1}\right.$ segment); $\mathrm{PCoA}=$ posterior communicating artery; proc. $=$ process; $\mathrm{SCA}=$ superior cerebellar artery; zyg. = zygomatic.

CN IV, $0.6 \mathrm{~mm}$ from the midline. The trigeminal motor nucleus limited the approach laterally, being located 6.3 $\mathrm{mm}$ from the midline.

A paramedian infracollicular area can be tailored between the projection of the facial nerve fibers on the facial colliculus and the superior limits of the nucleus of the hypoglossal nerve and the dorsal nucleus of the vagal nerve, extending a mean distance of $9.2 \mathrm{~mm}$ vertically and approximately $0.3 \mathrm{~mm}$ from the midline. ${ }^{37}$

\section{Median Sulcus of the Fourth Ventricle}

An approach through the midline, between the projection of the CN VI nuclei on the surface and the projection of the CN III nuclei on the midbrain surface, was proposed by Bricolo et al. ${ }^{5}$ taking advantage of the sparseness of crossing fibers (Fig. 2C). Even the slightest lateral retraction may provoke extraocular movement disorders caused by damage to the medial longitudinal fascicle.

\section{Medulla Oblongata}

\section{Anterolateral Sulcus}

Just lateral to the pyramid, the rootlets of the hypoglossal nerve leave the brainstem on the anterolateral sulcus. The short space between these rootlets and those of the C-1 nerve coincides with the decussation of the corticospinal tract. ${ }^{9}$ A paramedian oblique dissection may avoid the corticospinal tract and address lesions of the anterior lower medullary region (Fig. 3A and B).

\section{Posterior Median Sulcus}

A neurotomy on the median sulcus provides a corri- 

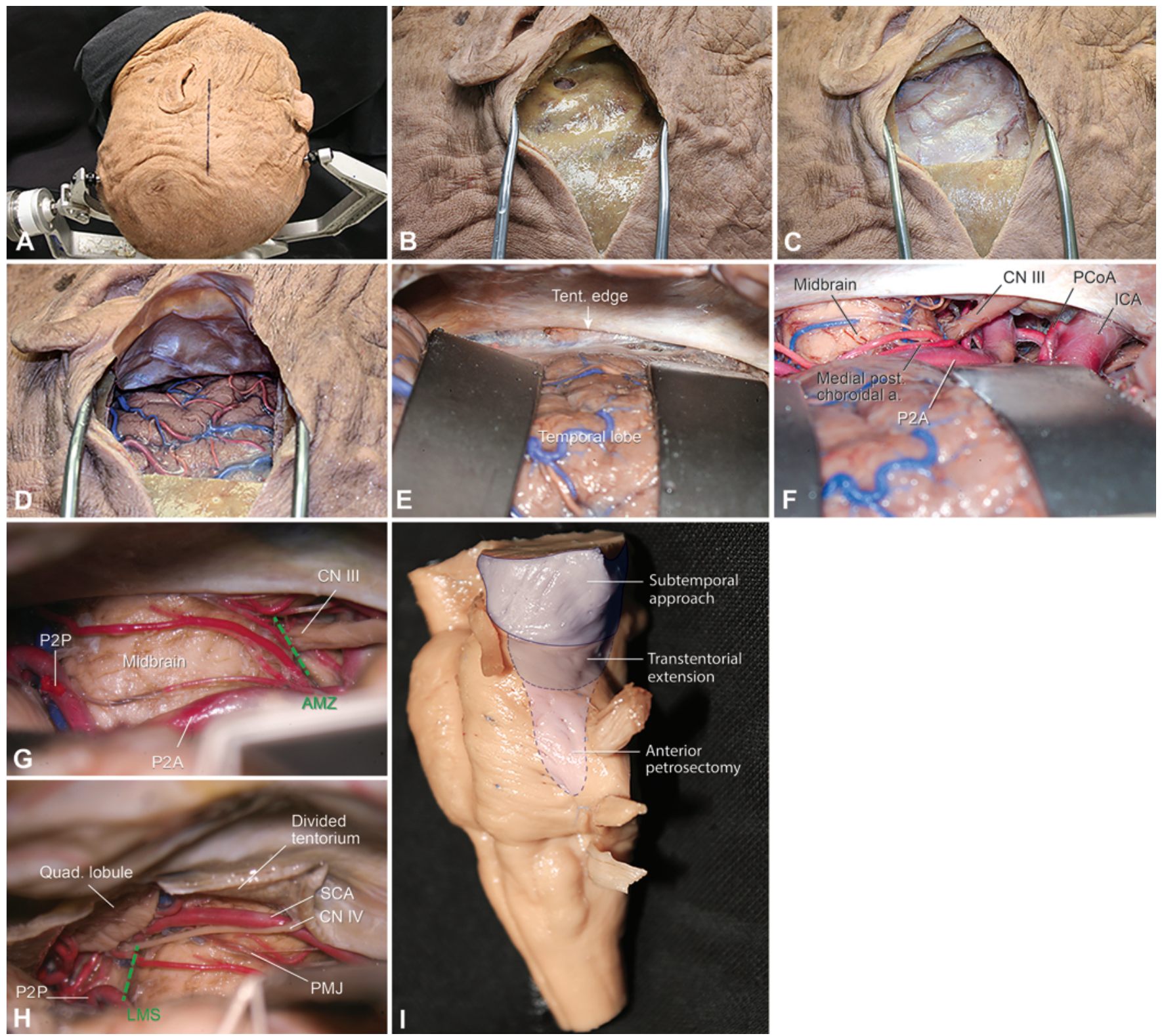

FIG. 5. Subtemporal approach. A: Photograph showing the site of skin incision. B: A bur hole is placed just above the root of the zygomatic arch. C: A square craniotomy is made extending two-thirds in front of the bur hole and one-third behind it. The inferior edge of the craniotomy is then drilled flush with the middle fossa floor. D: The dura is elevated, exposing the temporal lobe. E: The microdisection is carried between the basal surface of the temporal lobe and the tentorial edge, through the arachnoid of the ambient cistern. F: The area of exposure provided by this approach, including part of the anterior and the lateral incisural spaces, leads to the entire lateral midbrain surface. G: Magnified view of the lateral midbrain though the opened interpeduncular and ambient cisterns. This approach also exposes 2 safe entry zones: the anterior mesencephalic zone (AMZ; green dashed line) and the lateral mesencephalic sulcus (LMS; green dashed line seen in $\mathrm{H}$ ). H: Cutting the tentorium significantly improves visualization of the pontomesencephalic junction (PMJ) and the lateral upper pons. I: Progressive increases to a simple subtemporal approach in the area and length of exposure after adding a transtentorial extension and then an anterior petrosectomy. ICA = internal carotid artery; medial post. choroidal $\mathrm{a} .=$ medial posterior choroidal artery; $\mathrm{P} 2 \mathrm{~A}=$ anterior part of the $\mathrm{P}_{2}$ segment of the posterior cerebral artery; $\mathrm{PCOA}$ = posterior communicating artery; quad. = quadrangular; $\mathrm{SCA}=$ superior cerebellar artery; tent. = tentorial.

dor near the center of the medulla. ${ }^{5,9}$ Below the obex and restricted laterally by the clava, which covers the gracile nucleus, a surgeon can approach posteriorly placed lesions in a fashion similar to the traditional approach for intramedullary spinal cord lesions (Fig. 3C).

Olivary Zone

The olives are marked oval eminences on the antero- lateral surface of the medulla, limited medially by the anterolateral sulcus and the pyramids and posteriorly by the posterolateral sulcus. In a cross section at the level of the inferior olivary nucleus, fibers of the hypoglossal nerve separate it from the corticospinal tract running within the pyramids. The olives are also limited medially by hypoglossal nerve fibers and the medial lemniscus and are lim- 
ited posteriorly mainly by the tectospinal and spinothalamic tracts.

Recalde et al. ${ }^{35}$ identified a safe depth of dissection via the olive, ranging from 4.7 to $6.9 \mathrm{~mm}$, with a vertical length of $13.5 \mathrm{~mm}$ (Fig. 3).

\section{Lateral Medullary Zone "Inferior Cerebellar Pedun-} cle Approach"

Akin to the lateral pontine zone in the pons, the lateral medulla has recently been shown by Deshmukh et al. to be a relatively safe entry zone for resection of dorsolateral medullary lesions. ${ }^{14}$ These authors recently reported their experience with 4 patients whose lesions were approached through the foramen of Luschka with an incision in the inferior cerebellar peduncle and whose outcomes were excellent. ${ }^{14}$ We call the access through this approach the lateral medullary zone. Via a retrosigmoid approach, the foramen of Luschka is opened, and the origins of CNs IX and $X$ are identified. Then a small vertical incision is made in the inferior cerebellar peduncle inferior to the cochlear nuclei and posterior to the origin of CNs IX and X.

\section{Surgical Approaches}

\section{Orbitozygomatic}

For neurosurgeons to perform the orbitozygomatic approach, the patient is placed supine and the head is elevated above the level of the heart, rotated $15^{\circ}$ to $30^{\circ}$ contralaterally, and the neck is slightly extended (Fig. 4A). A hemicoronal skin incision is performed, the skin flap is reflected, and the galeal flap is dissected and prepared. An interfascial dissection is performed, exposing the zygomatic process of the frontal bone and the frontal process of the zygomatic bone (Fig. 4B). The temporal fascia is elevated, and a subperiosteal dissection is performed, revealing the zygomatic arch. The temporal muscle is then elevated, and a pterional craniotomy is tailored as proposed by Yaşargil ${ }^{39}$ (Fig. 4C). The orbitozygomatic osteotomy is then completed as a second step, returning the temporal muscle to its original position in a sequence of 6 cuts as modified by Zabramski et al. ${ }^{40}$ (Fig. 4D and E). The dura is opened with an arciform incision.

The sylvian fissure is opened, and the dissection is carried proximally, following the $\mathrm{M}_{1}$ segment of the middle cerebral artery toward the carotid and chiasmatic cisterns. These cisterns are opened, and the focus of the dissection is then turned to the carotico-oculomotor triangle, which is entered in order to reach the crural and interpeduncular cisterns. Their arachnoid trabeculae are also opened widely. Tracking up the oculomotor nerve to its entry zone leads the surgeon to the cerebral peduncle (Fig. 4F). Possible lesions emerging on the anterior surface of the midbrain, pontomesencephalic junction, and upper pons can be accessed (Fig. 4G). This approach also provides a straight path to the aforementioned anterior mesencephalic safe entry zone between the PCA and SCA, laterally to $\mathrm{CN}$ III (Fig. 4H). Clinically, the ventral approaches are often avoided because of the rich motor tracts that travel ventrally in the brainstem; nonetheless, in select cases, anterior approaches may be used to resect lesions in the midbrain (Fig. 4I-J).

Depending on the anteroposterior extension of the le- sion, as well as its closest point to the surface on the cerebral peduncle, a contralateral orbitozygomatic approach offers the best approach for complete resection of a midbrain lesion. Opening the Liliequist membrane allows the surgeon to reach the interpeduncular cistern, a very complex region demanding extremely delicate dissection. This small region is populated by the basilar artery bifurcation, both PCAs and SCAs, the medial posterior choroidal artery, and the thalamoperforating arteries and direct perforators of the proximal SCA, restricting surgical freedom significantly.

Lesions of the thalamomesencephalic junction are extremely challenging to expose. After widely dissecting the sylvian fissure, a small corridor is developed through the lenticulostriate arteries, between the posterior limits of both the gyrus rectus and the medial orbital gyrus, and the $\mathrm{M}_{1}$. We have described this approach-the transanterior perforating substance (TAPS) approach-previously, and it demands a small opening on the anterior perforating substance, posterior to the olfactory stria and near the optic tract. ${ }^{16}$

\section{Subtemporal}

For neurosurgeons to perform the subtemporal approach, the patient is placed in a lateral decubitus position, and the sagittal suture is kept parallel to the floor. A straight vertical incision is placed in front of the tragus, and the temporal fascia and muscle are opened in the same fashion, exposing mainly the temporal bone (Fig. 5A). A bur hole is made just above the root of the zygomatic arch, and the width of the craniotomy is tailored according to a preoperative plan for the best angle of approach to the brainstem (Fig. 5B and C). The dura is opened in a U-shaped fashion, and the dissection is carried below the base of the temporal lobe until the tentorial edge is exposed (Fig. 5D and E). The anterior and middle incisural spaces are then visible. The arachnoid of the ambient, crural, and interpeduncular cisterns is opened widely (Fig. 5F). The lateral surface of the midbrain, down to the pontomesencephalic junction, occupies most of the operative field, with the anterior $\mathrm{P}_{2}$ segment $\left(\mathrm{P}_{2} \mathrm{~A}\right.$ crural $)$ and $\mathrm{P}_{2} \mathrm{P}$ (ambient) segments of the PCA and the middle posterior choroidal artery crossing the field. The lateral mesencephalic vein frequently covers the lateral mesencephalic sulcus (Fig. 5G). This corridor yields an oblique access to the anterior mesencephalic zone and a straight route to the lateral mesencephalic sulcus (Fig. $5 \mathrm{H}$ and I). When possible, care must be exercised to avoid traversing the floor of the fourth ventricle. Approaches through the floor of the fourth ventricle can result in significant lower cranial nerve deficits and respiratory and swallowing difficulties.

\section{Subtemporal Transtentorial}

A transtentorial extension to the subtemporal approach is obtained by sectioning and retracting the tentorium just before the trochlear nerve pierces it. The pontomesencephalic junction and an anterolateral perspective of the upper pons can then be appreciated, in addition to the SCA running in the direction of the quadrigeminal cistern (Fig. $5 \mathrm{H})$. 


\section{Anterior Petrosectomy}

An anterior petrosectomy or Kawase approach can be added to the subtemporal approach or performed alone when aiming for a more anterolateral view of the pons from above in order to focus on lesions anterior to the trigeminal nerve (Fig. 5I). The petrous bone in front of the internal acoustic canal is drilled away; the exposure is limited anteriorly by the $\mathrm{V}_{3}$ segment of the trigeminal nerve and laterally by the greater superficial petrosal nerve.

\section{Suboccipital Telovelar}

For neurosurgeons to perform the suboccipital telovelar approach, the patient is placed in a prone position, the head is flexed, and a straight median incision is made to expose the myofascial layer, which is opened on the midline and retracted laterally (Fig. 6A). The suboccipital area is exposed together with the C-1 posterior arch. A median suboccipital craniotomy large enough to retract both cerebellar tonsils superolaterally is tailored using 2 bur holes, which are placed laterally at the superior edge (Fig. 6B and C). A laminoplasty of the $\mathrm{C}-1$ arch may be performed to significantly extend the vertical angle of approach. ${ }^{13}$

The dura is opened in a Y-shaped fashion to preserve the occipital sinus. Opening the dura exposes the cerebellar tonsils, which hide the cerebellomedullary fissure (Fig. 6D). By slightly retracting the tonsils and carefully pushing the telovelotonsillar segment of the posterior inferior cerebellar arteries laterally, it is possible to reach and open the tela choroidea. The tela choroidea, together with the inferior medullary velum, constitute the inferior half of the roof of the fourth ventricle (Fig. 6E). ${ }^{29}$ The telovelar junction attaches medially to the nodule and extends laterally into the lateral recess. Dividing the tela choroidea bilaterally is enough to bring the whole rhomboid fossa into the surgical field, as well as the lateral recesses (Fig. 6F). The safe zones above and below the facial colliculus are visible, as is the superior half of the median sulcus (Fig. 6G). Opening the inferior velum will expose the superior half of the ventricular roof beside the superolateral recesses.

\section{Median Supracerebellar Infratentorial}

The setting for the median supracerebellar infratentorial approach is very similar to the suboccipital telovelar approach described above, except that the skin incision is extended a bit cranially to ensure that the craniotomy extends to a point just above the transverse sinuses (Fig. 7A and B). Doing so allows the tentorium to be intermittently retracted. The dura is again opened in a Y-shaped fashion, and the dissection is begun via the tentorial surface of the cerebellum, opening the supracerebellar cistern and coagulating as few bridging veins as possible (Fig. 7C and D). The quadrigeminal cistern sits deeply in the dissection bed. It contains a complex venous structure that covers the pineal gland and includes the internal cerebral and internal occipital veins, the basal veins of Rosenthal, and the vein of the cerebellomesencephalic fissure (Fig. 7E and F). Continuing the dissection more deeply leads the neurosurgeon to the quadrigeminal plate, containing the superior and inferior colliculi and the intercollicular safe zone (Fig. 7G and $\mathrm{H}$ ). The pulvinar and the tentorial edge laterally limit the exposure of both the superior and inferior brachia.

\section{Extreme Lateral Supracerebellar Infratentorial}

For neurosurgeons to perform the extreme lateral supracerebellar infratentorial approach, the patient is placed in a lateral decubitus position, and the head is slightly flexed and rotated ipsilaterally. A retroauricular straight skin incision is made, the myofascial layer is elevated, and the flap is retracted anteriorly (Fig. 8A). A bur hole is placed just above the asterion on the parietomastoid suture, and a modified retromastoid craniotomy is tailored that extends across the transverse sinus, which eases surgical exposure through its intermittent retraction. The dura is opened using an inverted T-shaped incision, leaving a dural base along the transverse sinus and another along the sigmoid sinus. The dissection is made by cutting the arachnoid along the tentorial surface of the cerebellum, coagulating as few bridging veins as possible to reach the cerebellomesencephalic fissure (Fig. 8B).

The ambient cistern is then opened, exposing the trochlear nerve running along the posterolateral midbrain, frequently close to the SCA (Fig. 8C). Turning the focus to the midline and opening the quadrigeminal cistern provides an oblique approach to the collicular region, mainly to the inferior colliculus. The trochlear nerve can be traced to its origin laterally, below the inferior colliculus (Fig. 8D). Retracting the cerebellum closer to the transition between the tentorial and petrosal surfaces offers a more lateral perspective of the midbrain, centered on the lateral mesencephalic sulcus. Figure $8 \mathrm{E}$ reveals the window on the brainstem provided by this important approach. The supracerebellar infratentorial approach is a robust approach; a more superior view can be obtained by cutting the tentorium. This addition allows the surgeon to attack lesions in the medial temporal lobe and thalamus. Figure $8 \mathrm{~F}$ and $\mathrm{G}$ demonstrate a lesion resected via the extreme lateral supracerebellar infratentorial approach.

\section{Retrosigmoid}

For neurosurgeons to perform the retrosigmoid approach, the patient is placed in a lateral position with the head slightly flexed and rotated toward the ipsilateral side (Fig. 9A). A straight skin incision is placed 2 fingerbreadths behind the earlobe, exposing the myofascial layer. The myofascial layer is elevated, and the intersection of the parietal, temporal, and occipital bones is exposed using a self-retractor. One bur hole located just above the asterion on the parietomastoid suture is enough to perform a retromastoid craniotomy, with the anterior edge level with the posterior margin of the sigmoid sinus (Fig. 9B and $\mathrm{C}$ ). The dura is opened using an inverted T-shaped incision, leaving a dural base along the transverse and the sigmoid sinuses (Fig. 9D). The microsurgical dissection starts along the petrosal surface of the cerebellum, reaching the cerebellopontine cistern (Fig. 9E). Arachnoid strands around the superior petrosal vein and its tributaries as well as around the trigeminal, facial, and vestibulocochlear nerves are cut with regard to the trajectories of the SCA and anterior inferior cerebellar artery. It is then possible to expose the middle cerebellar peduncle and the lateral pons, which contain 3 safe zones around the $\mathrm{CN} \mathrm{V}$ entry zone (Fig. 9F and G), as described above. We use the retrosigmoid approach more commonly than any other ap- 

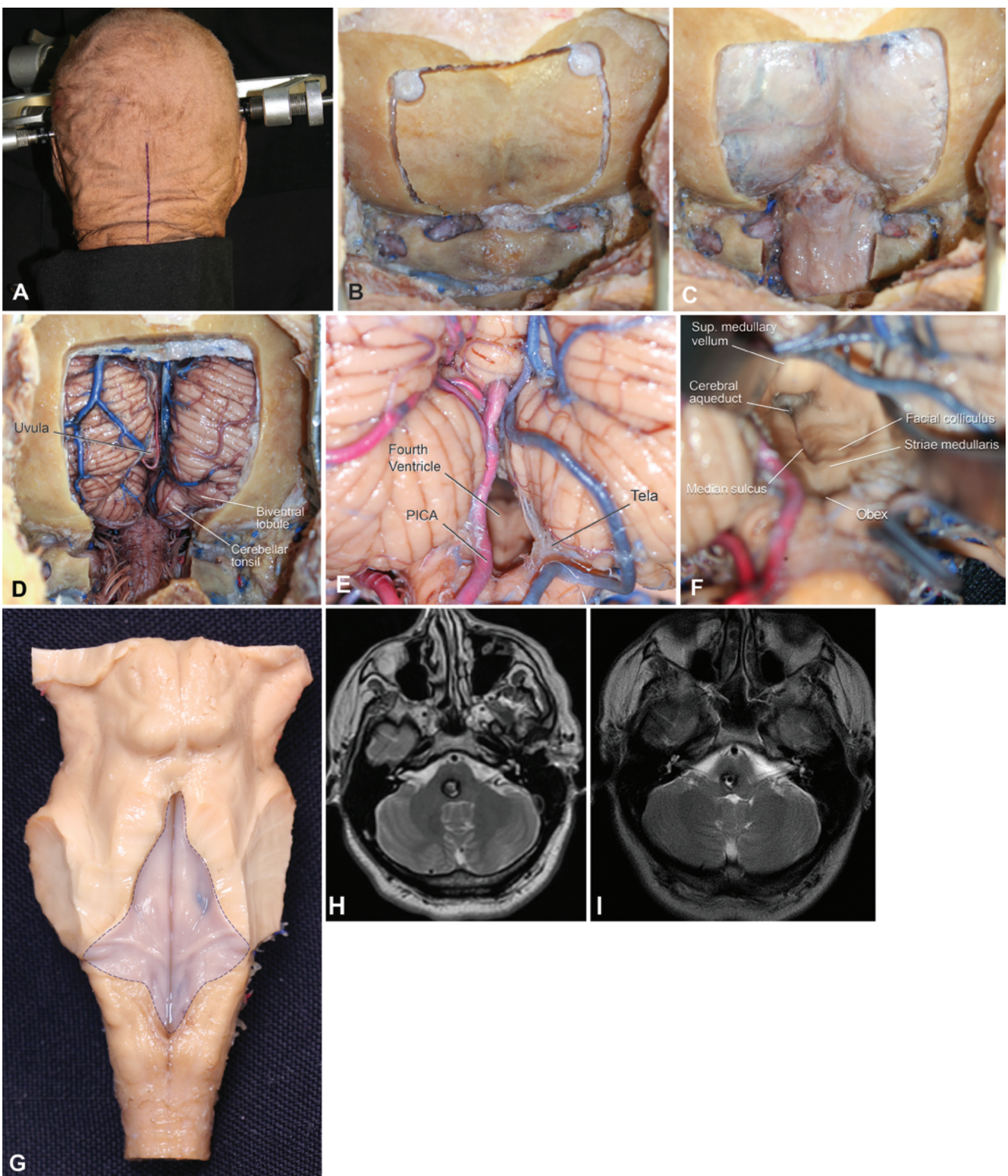

FIG. 6. Median suboccipital telovelar approach. A: A midline vertical skin incision is made. B: Two bur holes are made laterally to the inion just below the transverse sinuses, and a suboccipital craniotomy is tailored. C: The dura is exposed. The posterior arch of C-1 was opened to enhance the vertical angle of approach to the rhomboid fossa. D: The dura is opened, exposing cerebellar structures such as the cerebellar tonsils and the uvula, as well as the cerebellomedullary fissure. E: An opened foramen of Magendie, limited laterally by the tela choroidea, the tonsils, and the posterior inferior cerebellar arteries. F: Dissecting the tela choroidea and the inferior medullary velum elegantly exposes the whole rhomboid fossa and cerebral aqueduct, without the associated risks of splitting the vermis. G: The area on the dorsal brainstem exposed by the telovelar approach is represented by the shaded area. H: Preoperative T2-weighted MR image in this 55-year-old male reveals a hyperintense lesion surrounded by a hypointense hemosiderin halo in the dorsal pons. The lesion was approached via a suboccipital approach with gross-total resection of the lesion. I: Postoperative T2-weighted MR image reveals the resection cavity. PICA = posteroinferior cerebellar artery; sup. = superior. 

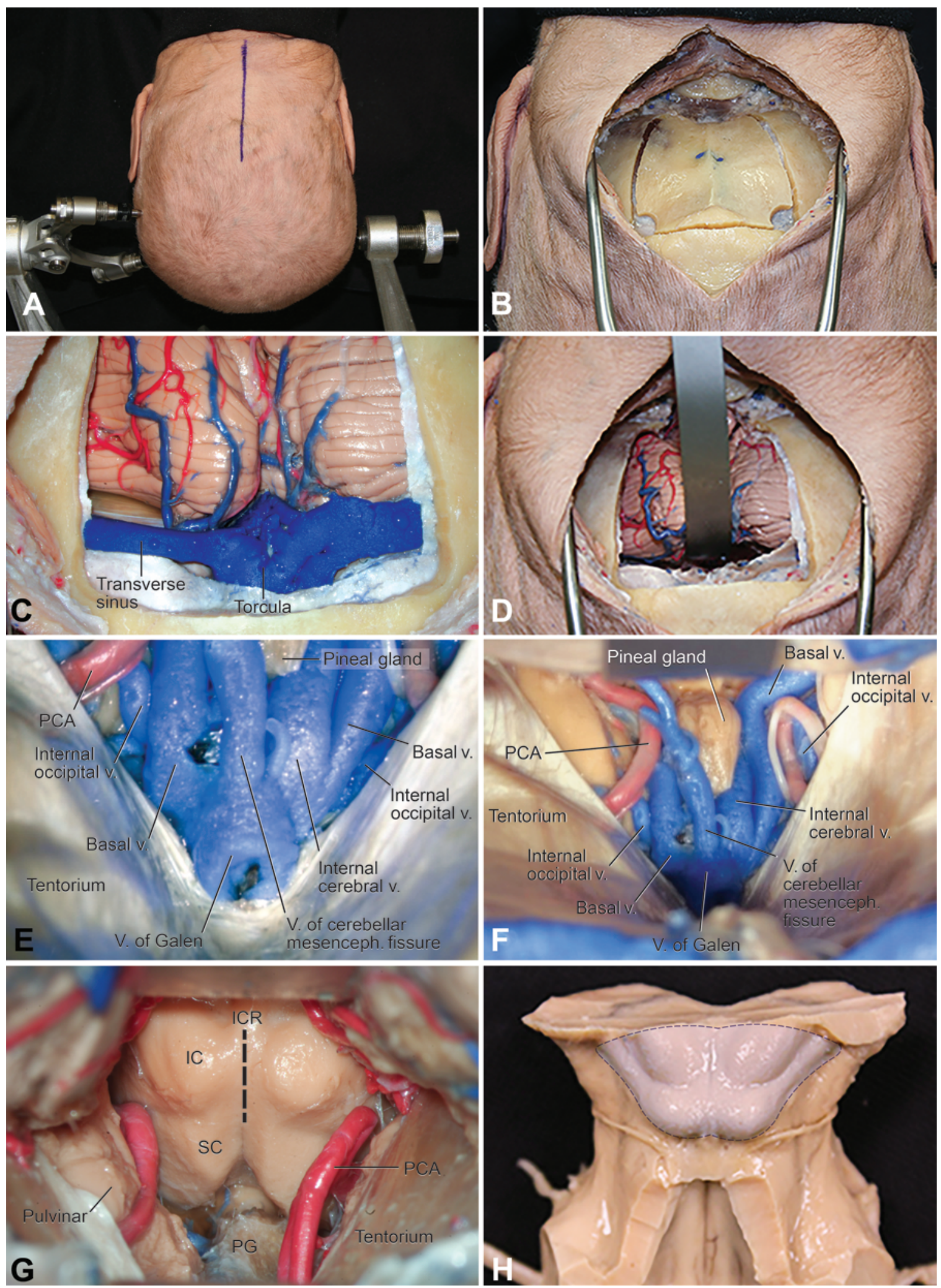

FIG. 7. Median supracerebellar infratentorial approach. A and B: The skin incision and craniotomy are extended farther cranially than in the median suboccipital approach to expose the transverse sinuses. C and D: After opening the dura in the customary Y-shape, the dissection is begun at the tentorial surface of the cerebellum. E: Most of the posterior incisural space is occupied by the vein of Galen complex. F: The pineal gland is exposed by dissecting downward. G: The objective is to expose the quadrigeminal plate below the pineal region at the base of the posterior incisural space. The intercollicular region (ICR) is shown in the center of the field. H: The shaded area represents the area of the dorsal brainstem exposed by this approach. $\mathrm{IC}=$ inferior colliculus; mesenceph. = mesencephalic; $\mathrm{PCA}=$ posterior cerebral artery; $\mathrm{PG}=$ pineal gland; $\mathrm{SC}=$ superior colliculus; $\mathrm{v}$. = vein . 

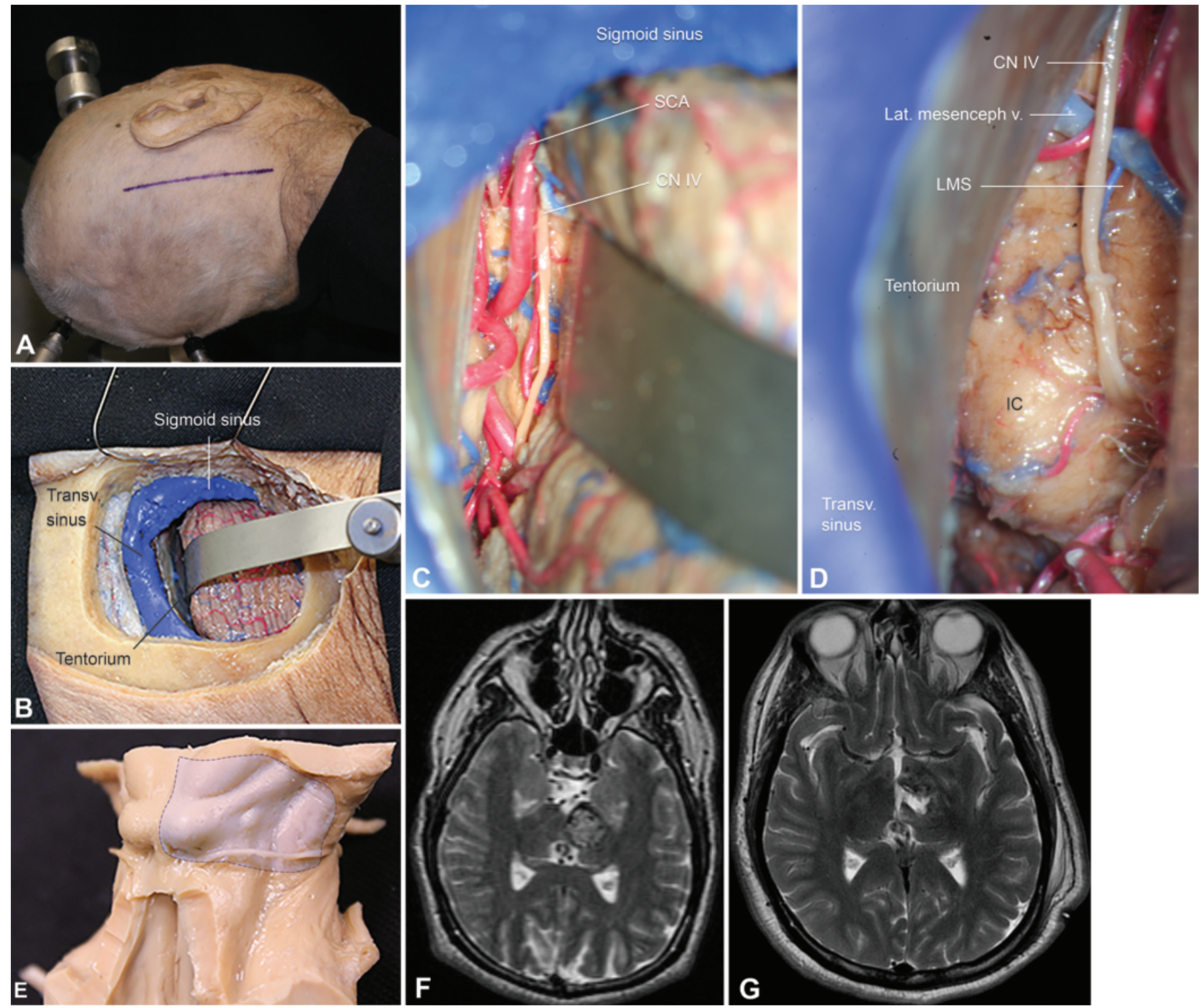

FIG. 8. Extreme lateral supracerebellar infratentorial approach. A: The patient is placed in a lateral position with the head fixed in a slight flexion and ipsilateral rotation. A straight incision is made in the retroauricular region. $\mathbf{B}$ : A retrosigmoid craniotomy is tailored to extend just above the transverse sinus; however, the dissection is carried superior to the tentorial surface of the cerebellum. C: After coagulating as few bridging veins as possible, and dissecting the arachnoid around the cerebellomesencephalic fissure and the superior petrosal vein, the neurosurgeon reaches the ambient cistern and the lateral segment of the quadrigeminal cistern. The trochlear nerve is seen crossing the cistern, along the superior cerebellar artery (SCA) and distal branches of the posterior cerebral artery. D: Magnified posterolateral view of the midbrain revealing the exit of the trochlear nerve inferolaterally to the inferior colliculus (IC). E: The shaded area corresponds to the area of exposure on the posterolateral brainstem gained by this approach. F and G: This 56-year-old male presented to our service with multiple hemorrhages attributable to this thalamomesencephalic cavernous malformation. F: Preoperative T2-weighted MR image reveals the cavernous malformation. The lesion was approached via an extreme lateral supracerebellar infratentorial approach, and the lesion was resected via the lateral mesencephalic sulcus. G: Postoperative MR image reveals gross-total resection of the lesion. Lat. mesenceph. v. = lateral mesencephalic vein; LMS = lateral mesencephalic sulcus; transv. = transverse.

proach. The posterolateral view afforded by this approach allows the surgeon to remove lesions while creating few motor deficits, albeit with significant, often temporary, sensory deficits (Fig. 9H-I). The use of an endoscope can allow surgeons to visualize the lateral pons without the need for the more extensive far-lateral approach.

\section{Far Lateral}

For the far-lateral approach, the patient is placed in the park-bench position, and a classic hockey-stick skin incision allows the surgeon to promptly identify each muscular layer down to the suboccipital triangle (Fig. 10A). ${ }^{24} \mathrm{~A}$ straight incision is faster but should be reserved for surgeons who are experienced with it. The very first layer exposed is composed of the sternocleidomastoid and trapezius muscles, which when retracted expose the splenius capitis muscle. The next layer comprises the longissimus capitis muscle laterally and the semispinalis capitis mus- 

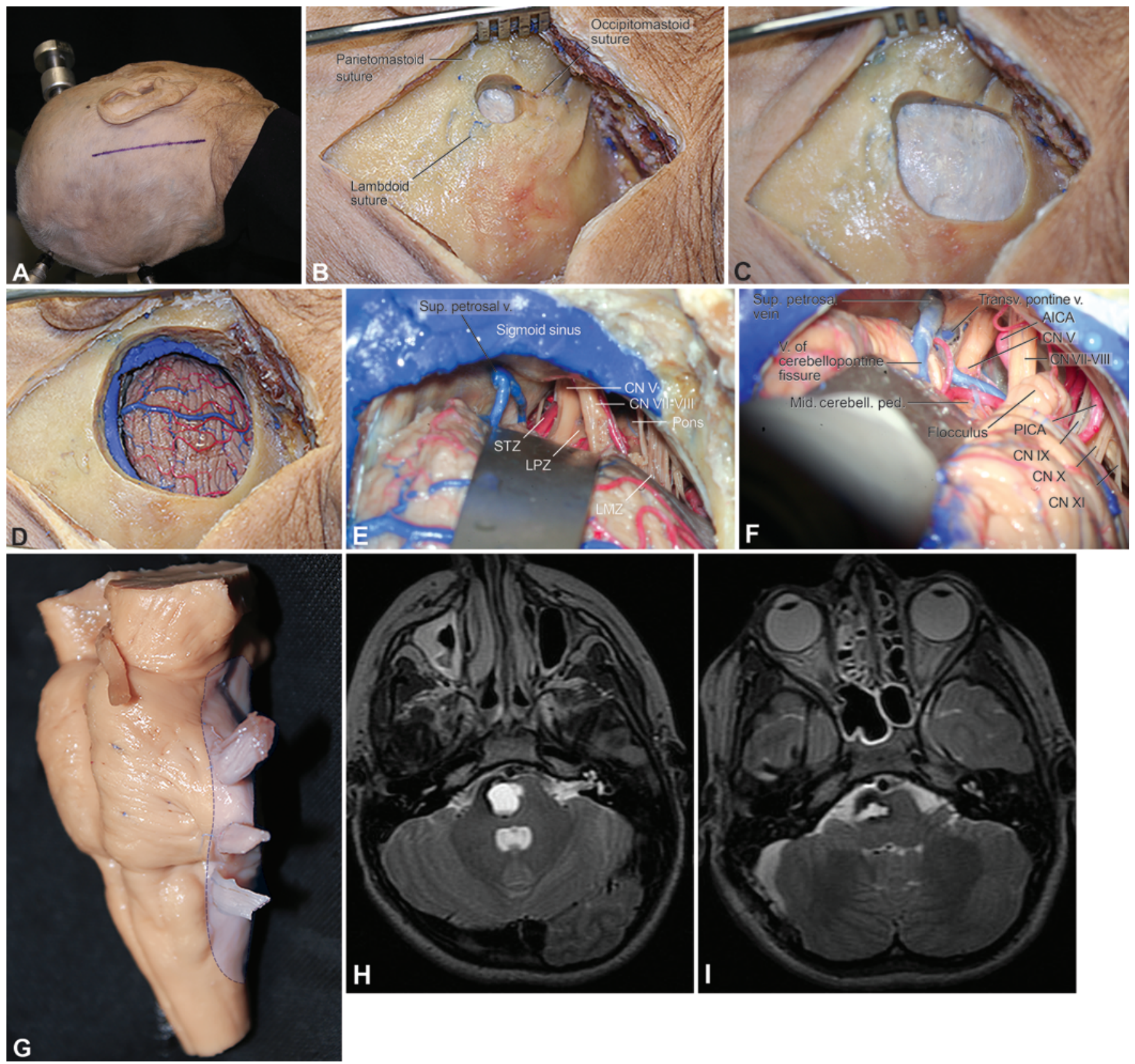

FIG. 9. Retrosigmoid approach. A: The cadaver head is placed in the lateral position. The skin incision is usually placed 2 fingerbreadths behind the pinna. B: A bur hole is drilled just cranial to the asterion, on the parietomastoid suture. C: The craniotomy is started with a C-shaped sawing line. The posterior edge of the sigmoid sinus as well as the transverse-sigmoid junction is skeletonized. D: The dura is opened and the cerebellar surface is exposed. E: The microsurgical dissection starts along the petrosal surface of the cerebellum, reaching the cerebellopontine cistern. The approach also provides access to the supratrigeminal (STZ) and the lateral pontine (LPZ) safe zones but provides a suboptimal angle of attack for the peritrigeminal zone. F: Lesions abutting the middle cerebellar peduncle or the lateral pontine surface can be resected using this route. G: The shaded area represents the area of exposure on the lateral brainstem produced by the retrosigmoid approach. $\mathrm{H}$ and $\mathrm{l}$ : This pontomedullary cavernous malformation in a 10-year-old male was approached via a retrosigmoid approach through the lateral medullary zone. $\mathrm{H}$ : Preoperative T2-weighted MR image demonstrates the lesion. I: Postoperative T2-weighted MR image reveals gross-total resection of this lesion. $\mathrm{AICA}=$ anteroinferior cerebellar artery; $\mathrm{LPZ}$ = lateral pontine zone; mid. cerebell. ped. = middle cerebellar peduncle; PICA = posterior inferior cerebellar artery; STZ = supratrigeminal zone; sup. = superior; transv. = transverse; $v$ = vein.

cle, which when elevated bring the suboccipital triangle into the surgical field.

The superior oblique, inferior oblique, and rectus capitis posterior major muscles limit the triangle that protects the $\mathrm{V}_{3}$ segment of the vertebral artery (Fig. 10B). The rectus capitis posterior minor muscle is located medially to the rectus capitis posterior major muscle. Both of these muscles are elevated, exposing the C-1 posterior arch and the vertebral artery (Fig. 10C). The ipsilateral half of the C-1 posterior arch is removed after subperiosteal dissection of 

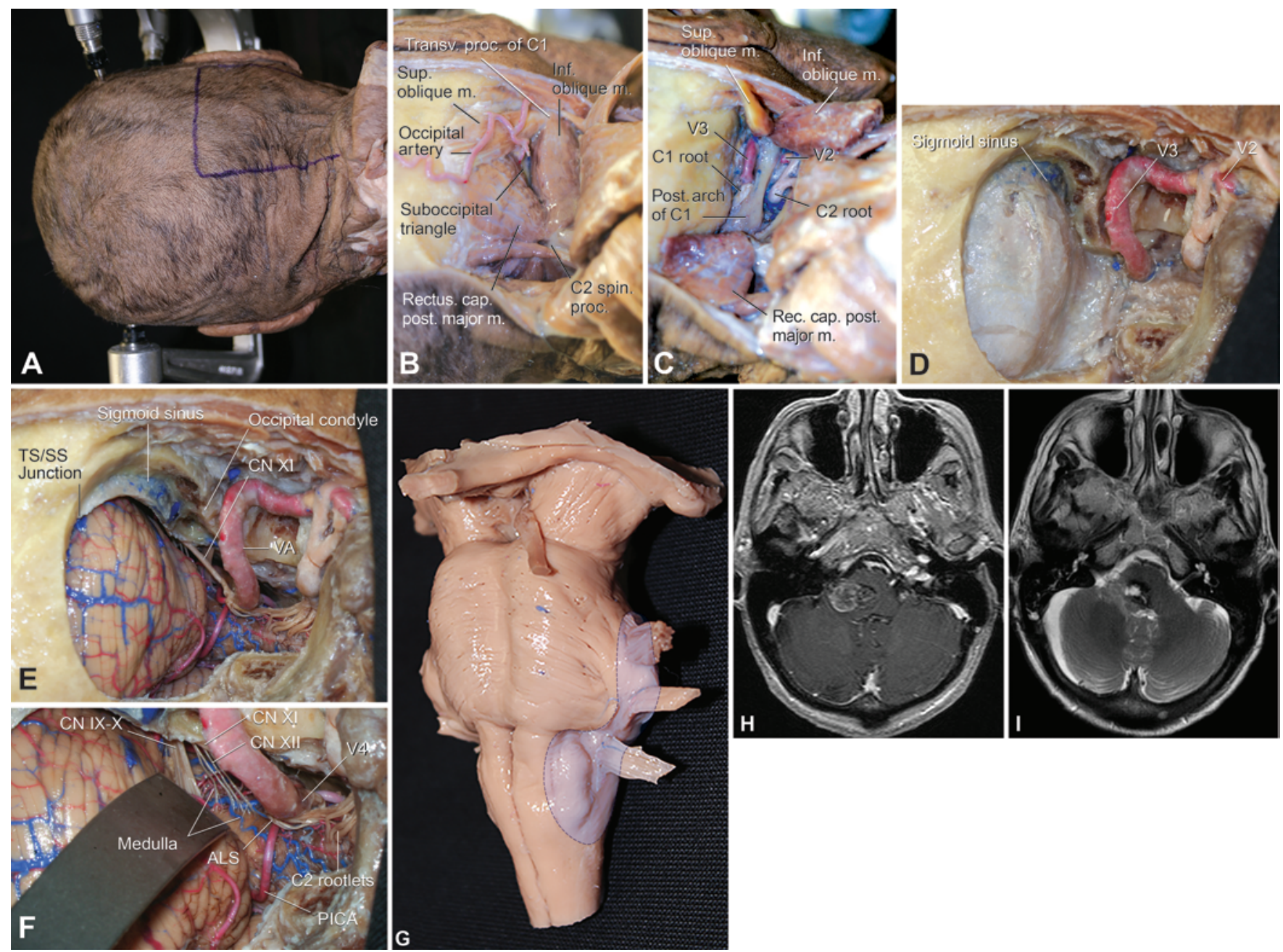

FIG. 10. Far-lateral approach. A: The classic hockey-stick incision with the patient in the park-bench position. B and C: A meticulous muscle dissection exposes the suboccipital triangle, covering the $V_{3}$ segment of the vertebral artery (VA). D: A lateral suboccipital craniotomy and a C-1 laminotomy. The posterior root of the transverse foramen may be drilled away to mobilize the vertebral artery, a step not required for most brainstem lesions. E: The posterior third of the occipital condyle may also be drilled away, widening the angle of attack for anterolateral medullary lesions. The dura is then opened. F: The posterolateral medulla is exposed for access to superficial lesions, including exposure of the trajectory of the lower cranial nerves to the jugular foramen, the vertebral artery, and posterior inferior cerebellar artery. The cervicomedullary junction is also exposed. Changing the angle of the microscope allows visualization of the anterolateral surface of the medulla. G: The shaded area correlates with the area of exposure on the brainstem accessed by the far-lateral approach. H: T1-weighted postcontrast MR image of the brain in a 67-year-old female reveals a lesion consistent with a cavernous malformation. The patient underwent a right far-lateral approach and resection of the lesion via the lateral pontine zone. I: Postoperative T2-weighted MR image reveals gross-total resection of the lesion. ALS = anterolateral sulcus safe zone; $\mathrm{C} 2$ spin. proc. = C-2 spinous process; inf. oblique $\mathrm{m} .=$ inferior oblique muscle; $\mathrm{PICA}=$ posterior inferior cerebellar artery; post. = posterior; rectus cap. post. major $\mathrm{m}$. = rectus capitis posterior major muscle; sup. oblique $\mathrm{m}$. = superior oblique muscle; transv. proc. of $\mathrm{C} 1=$ transverse process of $\mathrm{C}-1$; TS/SS junction $=$ transverse sinus/sigmoid sinus junction; $\mathrm{V} 2=\mathrm{V}_{2}$ segment of the vertebral artery; $\mathrm{V} 3=\mathrm{V}_{3}$ segment of the vertebral artery; $\mathrm{V} 4=\mathrm{V}_{4}$ segment of the vertebral artery.

the vertebral artery. The posterior root of the transverse foramen may be drilled away to mobilize the vertebral artery, although this is not required for most brainstem lesions.

A lateral suboccipital craniotomy is then tailored to the craniocaudal extension of the lesion, exposing the posterior margin of the sigmoid sinus (Fig. 10D). The posterior third of the occipital condyle may also be drilled away, preserving the hypoglossal canal and improving the angle of attack for lesions in the anterolateral medulla. The dura is then opened in a curvilinear fashion, starting close to midline and curving toward the transverse sigmoid junction (Fig. 10E).

The arachnoid over the cisterna magna is opened, as well as the lateral cerebellomedullary cistern. The $\mathrm{V}_{4}$ segment of the vertebral artery can be seen coursing laterally to the spinal accessory nerve and in front of the hypoglossal rootlets, where the posterior inferior cerebellar artery arises (Fig. 10F). A wide window is opened to reach the posterolateral medullary surface and the cervicomedullary junction. The accessory nerve, together with the glossopharyngeal and the vagus, enter the jugular foramen. 
The arachnoid covering the cerebellopontine cistern can be divided to approach the pontine surface. Continuing the dissection ventrally leads to the premedullary cistern, allowing access to the anterolateral sulcus and the olivary zone (Fig. 10G). Figure $10 \mathrm{H}$ and I illustrates removal of a lesion via the far lateral approach.

\section{Retrolabyrinthine}

For neurosurgeons to perform the retrolabyrinthine approach, the patient can be positioned supine using a thick roll under the ipsilateral shoulder, rotating the head contralaterally and flexing it. The surgery starts with a retroauricular C-shaped incision, followed by elevation and anterior retraction of the myofascial flap to expose the spine of Henle and the posterior margin of the external acoustic canal (Fig. 11A). Macewen's triangle is a landmark for identifying the mastoid antrum $1.5 \mathrm{~cm}$ below the surface and is limited superiorly by the suprameatal crest, anteroinferiorly by a line running along the superior and posterior margins of the external auditory canal and crossing the spine of Henle, and posteriorly by a tangential line from the posterior margin of the canal crossing the first line.

A mastoidectomy is the first step to this transpetrosal presigmoid approach. The suprameatal crest is the superior limit for the initial drilling, the posterior wall of the canal is the anterior limit, the mastoid tip is the inferior limit, and the sigmoid sinus is the posterior limit. The drilling should start by drawing an inverted $\mathrm{L}$ over the cortical bone, exposing the first air cells, and employing a uniform depth of dissection (Fig. 11B). The next objective is to reach the mastoid antrum cranially and expose the Trautmann's triangle caudally (Fig. 11C). This triangle represents an area of the posterior fossa dura limited by the sigmoid sinus posteriorly; the jugular bulb inferiorly; and the superior petrosal sinus, middle fossa dura, and otic capsule superiorly (Fig. 11D).

The dura is opened along the sinuses and retracted anteriorly. The petrosal surface of the cerebellum is initially exposed; however, after cerebrospinal fluid is drained from the cerebellopontine cistern, the surgeon can observe the flocculus behind the CN VII/VIII complex and the anterior inferior cerebellar artery (Fig. 11E and F). After dissecting the cistern widely, the surface of the middle cerebellar peduncle and the root entry zone of the trigeminal nerve can be seen (Fig. 11F). Its smaller motor root exits the pons superomedial to the larger sensory root. This approach provides a significantly more lateral approach to the supratrigeminal, peritrigeminal, and lateral pontine safe zones (Fig. 11G).

\section{Discussion}

The brainstem had been considered an untouchable region for decades, protected anteriorly by the clivus, laterally by the petrous bones, posteriorly by the cerebellum, and superiorly by the diencephalon. Because it harbors significant cranial nerve nuclei and tracts in a small sectional area, any manipulation can cause an elevated risk of morbidity.

Almost concurrently, Pool in $1968^{31}$ and Lassiter et al. in $1971^{25}$ reported their pioneering surgical series that offered a surgical option for managing intrinsic brainstem pathology. The following decades were marked by strong discussions on the indications and feasibility of brainstem surgery. But the expertise gained by select groups of neurosurgeons using an essential combination of progress in microsurgical instruments and techniques, skull base surgery, surgical planning with magnetic resonance imaging and image guidance, neuroanesthesia and neurointensive care, and intraoperative monitoring produced significantly better results and outcomes for patients with brainstem lesions. For instance, Bricolo et al. ${ }^{5,6}$ have published extensive work on brainstem tumors, introducing and discussing different safe entry zones. Similarly, the senior author of this paper (R.F.S.) has published a number of studies on the evolution of surgery for cavernous malformations of the brainstem..$^{1,11,32}$ Finally, other select groups have contributed meaningful additions to the surgical management of brainstem hemangioblastomas..$^{38,41}$

Experience in multiple surgical routes is key to selecting the right approach to different locations within the brainstem. A detailed review of the lesion on thin-cut T1-, T2-, and susceptibility-weighted MRI sequences is required. Image guidance supports anatomical and radiological knowledge, ensuring a perfect trajectory to the lesion. Nevertheless, whenever lesions do not rise to the pial or ependymal surface, it is essential to have a fundamental understanding of the concept of safe entry zones. Such landmarks have been described in surgical series and neuroanatomy studies (Tables 1 and 2). They represent small regions between vital neural structures and take advantage of the sparseness of perforators. ${ }^{2,5,20,23}$ Moreover, manipulation through these corridors is believed to minimize deficits when performed by experienced surgeons.

It is important to note that large intrinsic lesions may

TABLE 1. Surgical approaches to the brainstem according to lesion location

\begin{tabular}{llll}
\hline $\begin{array}{c}\text { Lesion } \\
\text { Location }\end{array}$ & \multicolumn{1}{c}{ Anterior } & \multicolumn{1}{c}{ Lateral } & Posterior \\
\hline Midbrain & OZ, mini-OZ, PT & Anterolateral: OZ, mini-OZ, ST & Median SCIT \\
\hline & & Posterolateral: paramedian or extreme lateral SCIT & \\
\hline Pons & ST \pm TT \pm AP, RL, RS & RS & SOTV \pm C-1 laminoplasty \\
\hline Medulla & FL & Upper Medulla: FL, RS & SOTV \\
\hline & & Lower Medulla: FL & \\
\hline
\end{tabular}

$\mathrm{AP}=$ anterior petrosectomy; $\mathrm{FL}$ = far lateral; $\mathrm{OZ}$ = orbitozygomatic; $\mathrm{PT}$ = pterional; $\mathrm{RL}$ = retrolabyrinthine; $\mathrm{RS}$ = retrosigmoid; $\mathrm{SCIT}=$ supracerebellar infratentorial; SOTV = suboccipital telovelar; $\mathrm{ST}$ = subtemporal; $\mathrm{TT}$ = transtentorial. 

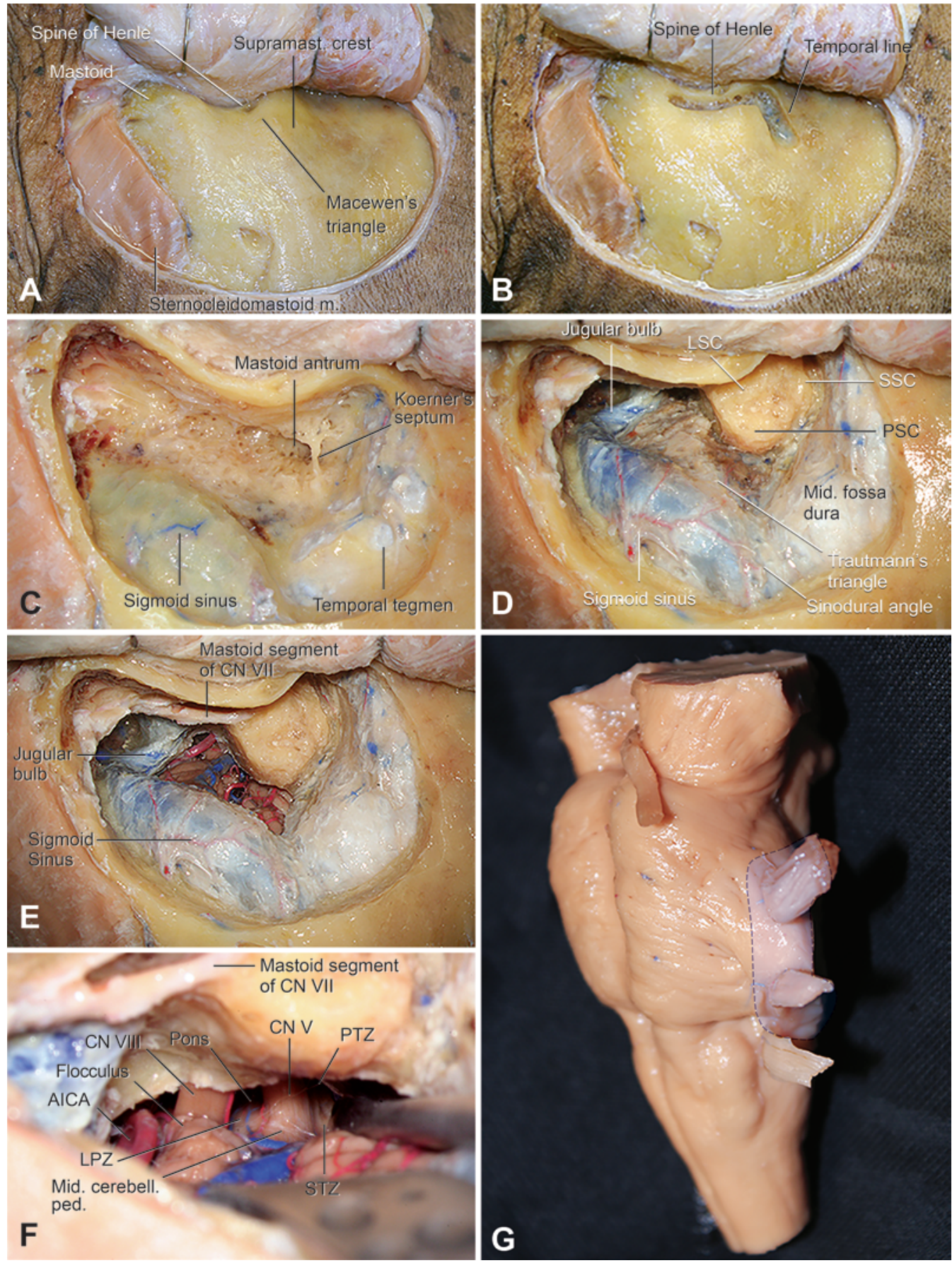

FIG. 11. Retrolabyrinthine approach. A: The patient is positioned supine, and the head is rotated toward the contralateral side. A retroauricular $\mathrm{C}$-shaped incision is placed on the left side; the skin flap is reflected forward. Exposure of the lateral surface of the mastoid bone with its landmarks is shown. B: Initial mastoid cortical drilling initiated just posterior to the spine of Henle. The mastoidectomy starts by drilling a straight cut along the temporal line aiming toward the sinodural angle. A second perpendicular line is drilled from the initial point toward the mastoid tip, bounded by the external auditory canal anteriorly. C: The mastoid cortex was removed and the air cells are open. Koerner's septum is thinned, unveiling the mastoid antrum, which is the largest mastoid cell. D: The semicircular canals are identified. The dura in front of the sigmoid sinus and above the jugular tubercle delineates Trautmann's triangle, E: The posterior fossa dura is then opened, taking into consideration the anatomy of the endolymphatic sac. The petrosal surface of the cerebellum comes to view, with the flocculus behind the CN VII/VIII complex. The distal anterior inferior cerebellar artery is also noted in relation to such complex. F: This presigmoid approach provides a straighter route to the lateral surface of the pons, although it results in a limited view and requires time-consuming drilling. The superior and middle neurovascular complexes of the cerebellopontine angle are exposed. The trigeminal nerve root entry zone determines 3 safe entry zones on the lateral pons: the lateral pontine (LPZ), peritrigeminal (PTZ), and supratrigeminal (STZ) zones. G: The area of exposure on the lateral pons that is provided by the retrolabyrinthine approach is shown by the shaded area. LSC $=$ lateral semicircular canal; $\mathrm{m}$. = muscle; mid. = middle; mid. cerebell. ped. = middle cerebellar peduncle; $\mathrm{PSC}=$ posterior semicircular canal; $\mathrm{SSC}=$ superior semicircular canal; supramast. $=$ supramastoid. 
TABLE 2. Accessible safe entry zones by surgical approach

\begin{tabular}{ll}
\hline \multicolumn{1}{c}{ Approach } & \multicolumn{1}{c}{ Safe Entry Zones } \\
\hline Orbitozygomatic & $\mathrm{AMZ}$ \\
\hline Subtemporal & $\mathrm{AMZ}$ \\
\hline Subtemporal transtentorial & $\mathrm{AMZ}, \mathrm{STZ}$ \\
\hline Anterior petrosectomy & $\mathrm{AMZ}, \mathrm{STZ}, \mathrm{PTZ}$ \\
\hline Suboccipital telovelar & $\mathrm{MS}$ \\
\hline Median SCIT & $\mathrm{LMS}, \mathrm{IC}, \mathrm{SC}, \mathrm{IF}$ \\
\hline Extreme lateral SCIT & $\mathrm{LMS}, \mathrm{IC}, \mathrm{SC}, \mathrm{IF}$ \\
\hline Retrosigmoid & $\mathrm{LMS}, \mathrm{STZ}, \mathrm{PTZ}, \mathrm{LPZ}, \mathrm{AL}, \mathrm{PM}, \mathrm{LMZ}$ \\
\hline Far lateral & $\mathrm{AL}, \mathrm{PM}, \mathrm{LMZ}$, olivary \\
\hline Retrolabyrinthine & $\mathrm{LMS}, \mathrm{STZ}, \mathrm{PTZ}, \mathrm{LPZ}, \mathrm{AL}, \mathrm{PM}, \mathrm{LMZ}$, \\
& olivary \\
\hline
\end{tabular}

$\mathrm{AL}=$ anterolateral sulcus of medulla; $\mathrm{AMZ}=$ anterior mesencephalic zone; $\mathrm{IC}$ = intercollicular; IF = infracolliclular; LMS = lateral mesencephalic sulcus; LMZ = lateral medullary zone; $\mathrm{LPZ}=$ lateral pontine zone; $\mathrm{MS}=$ median sulcus of fourth ventricle; $\mathrm{PM}=$ posterior median sulcus of medulla; $\mathrm{PTZ}=$ peritrigeminal zone; SC = supracollicular; SCIT = supracerebellar infratentorial; STZ = supratrigeminal zone.

distort the present surgical windows and their landmarks and safe entry zones provided by each approach.

\section{Midbrain Lesions}

Both pterional and orbitozygomatic approaches provide a straightforward route to the ipsilateral cerebral peduncle (Table 1). The orbitozygomatic approach offers the widest vertical angle of attack and surgical freedom in the peduncular region. Lesions surfacing anteriorly on the peduncle, pontomesencephalic junction, or upper pons can be managed through these approaches. The anterior mesencephalic zone can also be nicely exposed when neither color nor volume alteration is visible on the pial surface. We currently use keyhole approaches rather than extensive dissections and large craniotomies whenever possible. The mini-modified orbitozygomatic (mini-OZ) approach generally replaces full orbitozygomatic craniotomies. The mini-OZ requires a significantly smaller skin incision, just behind the hairline, and the bone opening is reduced to a $3-\mathrm{cm}$ bone piece, starting just lateral to the supraorbital notch and extending to a point just lateral to the frontozygomatic suture (Fig. 12). Our group described the same anatomical exposure when quantitatively comparing the mini-OZ, pterional, and orbitozygomatic approaches..$^{18}$ Another interesting option is the transciliary supraorbital approach, which utilizes a discreet eyebrow incision and provides a similar bone opening and corridor as that of the mini-OZ (Fig. 12 inset). ${ }^{10}$

The subtemporal approach exposes the entire lateral surface of the midbrain. With proper planning, the craniotomy will provide an adequate anterior or posterior view of the middle incisural space. However, the extreme lateral supracerebellar infratentorial approach provides an angled view of the posterolateral surface of the midbrain, without the drawbacks of a posterior subtemporal microdissection and retraction. The 2-point method may aid such decision making when approaching lesions on the midbrain tegmentum. ${ }^{7}$ Lesions that are deeper and closer to the lateral surface should be approached through the lateral mesen-

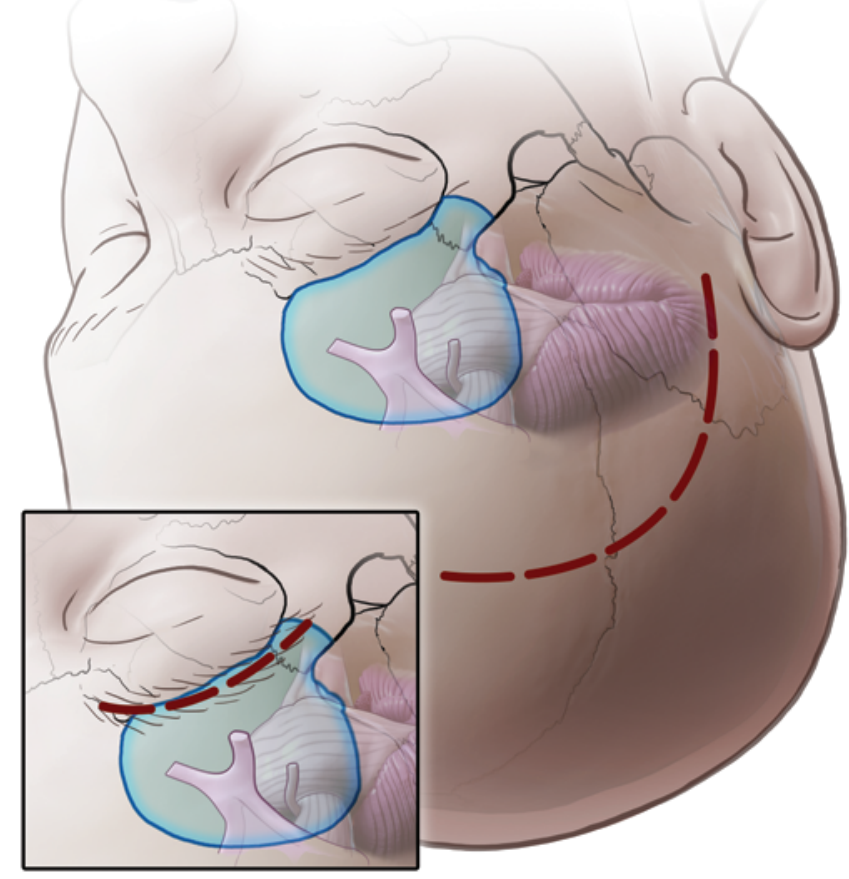

FIG. 12. Keyhole alternatives to the classic orbitozygomatic (OZ) approach to the brainstem, which demand less myofascial dissection and provide a significantly smaller bony opening but similar exposure of the basal cisterns compared with the classic OZ approach. In these 2 alternatives, 2 different skin incisions can be used, but the bony openings are essentially the same. The mini-modified OZ (mini-OZ) approach is shown with its reduced skin incision; the inset illustration shows the transciliary supraorbital approach with its discreet eyebrow incision. Reproduced with permission from Barrow Neurological Institute, Phoenix, Arizona.

cephalic sulcus. The long axis of the lesion will dictate whether the subtemporal or extreme lateral supracerebellar infratentorial corridors are used. Lesions within the tegmentum that are closer to the ventral surface may be best approached through the anterior mesencephalic zone via a pterional or orbitozygomatic approach.

Finally, tectal plate lesions may be reached through any variation of the supracerebellar infratentorial approach, depending on where the lesion is closest to the pial surface. ${ }^{12}$ The median supracerebellar infratentorial approach is employed for midline lesions or when a dissection through the intercollicular region is necessary. The extreme lateral approach is reserved for lateral lesions on the tectal plate that occur on the surface of either the superior or inferior colliculus. This approach provides a wide horizontal angle of approach to any lesion on the posterolateral midbrain surface including the lateral mesencephalic sulcus. The exit of the trochlear nerve is an important landmark; dissecting this nerve within the ambient cistern medial to its exit within the quadrigeminal cistern will lead to the inferior colliculus, which is superomedially placed.

\section{Pontine Lesions}

As previously mentioned, the pterional and the orbitozygomatic approaches offer some exposure of the ventral upper pons. The transtentorial extension of the subtempo- 
ral approach allows exposure of the lateral upper pons as well as the pontomesencephalic junction. However, the trigeminal nerve entry zone is actually the major landmark in approaching most of the anterolateral pontine lesions. Thus, approaches that aim toward the emergence of $\mathrm{CN}$ $\mathrm{V}$ represent the main routes to managing pontine lesions.

Despite the complexity of dissection and drilling work, the anterior petrosectomy offers the best direct anterior view of the peritrigeminal zone. The retrolabyrinthine approach is also time consuming and provides a limited posterolateral view of the $\mathrm{CN} \mathrm{V}$ entry zone. However, the retrosigmoid approach is the workhorse for managing anterior and lateral pontine and high-riding medullary lesions, while providing a more posterolateral exposure of the pons and the middle cerebellar peduncle than other approaches (Fig. 13). Additionally, its widespread use in managing vestibular schwannomas and neurovascular conflicts provides experience for those who employ it to attack pontine lesions directly or through the peritrigeminal, supratrigeminal, and lateral pontine safe zones.

Dorsal pontine lesions abutting or close to the rhomboid fossa can be reached through a suboccipital telovelar approach. The depth of the rhomboid fossa is rich in nuclei and tracts, which limits free manipulation. The facial colliculus is the major landmark and guides the surgeon to the supracollicular and infracollicular safe zones when the lesion does not reach the surface. Lastly, cranial lesions can be resected through the median sulcus.

\section{Medullary Lesions}

The far-lateral approach is chosen to manage anterolateral lesions within the medulla. Cranial lesions not abutting the pial surface are reached through the olivary safe
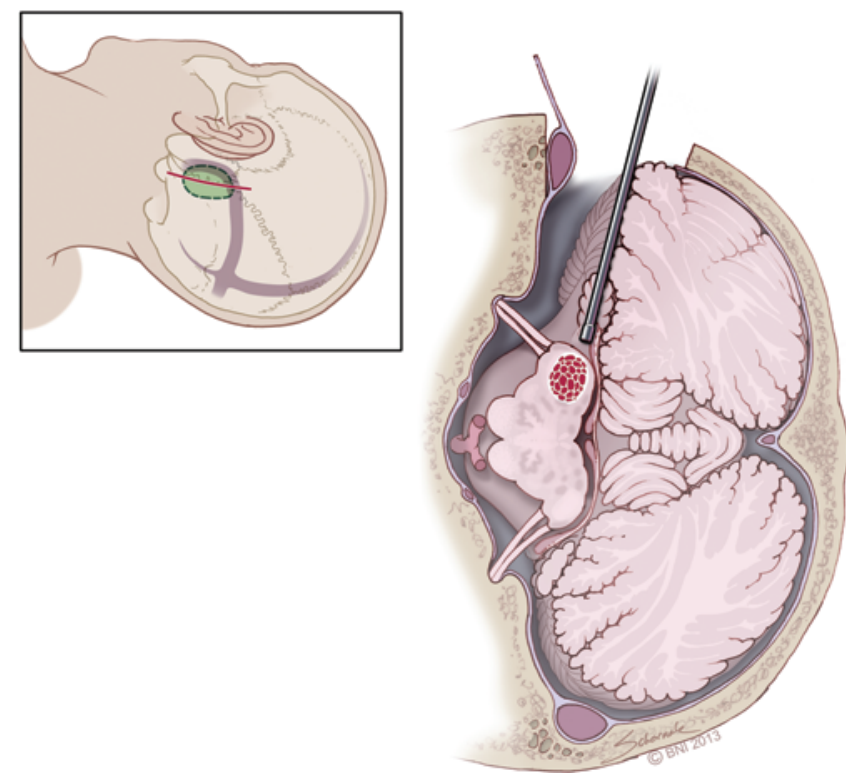

FIG. 13. Illustration of a retrosigmoid craniotomy and microsurgical resection of an intrinsic lesion in the posterolateral medulla. The flocculus and choroid plexus protruding through the foramen of Luschka are mobilized, and the lateral medullary zone is entered to gain access to the lesion. Reproduced with permission from Barrow Neurological Institute, Phoenix, Arizona. First published in Deshmukh et al: J Neurosurg 121:723-9, 2014. zone. Otherwise, caudal lesions are approached through the anterolateral sulcus between the emergence of the hypoglossal and C-1 spinal nerve rootlets.

Lateral lesions close to the pontomedullary junction require mainly a retrosigmoid approach. This craniotomy can also be employed to manage some lateral caudal lesions if the long axis of the lesion is amenable to the oblique angle of view provided by the retrosigmoid approach (Fig. 13). ${ }^{4}$ Deeper dorsolateral lesions can be managed by tailoring an incision over the lateral medullary zone. Finally, posterior lesions can be directly accessed via a median suboccipital approach. The neurosurgeon should avoid any manipulation on the calamus scriptorius, an extremely eloquent region, populated by the nuclei of the lower cranial nerves. A posterior midline medullary incision below the obex is advised instead.

\section{A Note of Caution}

Brainstem lesions represent some of the most challenging entities faced by neurosurgeons. In this article, we have summarized the approaches and safe entry zones into the brainstem. However, no region in the brainstem is truly "safe" for entry. The zones presented in this paper represent the regions through which lesions can be accessed with the least morbidity. As clinical experience from our group has demonstrated, surgery in the brainstem is associated with a high rate of temporary, but real, deficits. ${ }^{1}$ Practitioners with limited experience operating in the posterior fossa and the brainstem should consider referring these patients to highvolume centers with the requisite experience.

\section{Conclusions}

Progress in medical technology combined with the experience and dissemination of knowledge regarding microsurgery and brainstem pathology changed the paradigm that the brainstem represented a "no man's land" for neurosurgeons.

Our revisit of the main safe entry zones provided by each major surgical approach to the brainstem, which includes detailed dissection images and shows areas of exposure, may allow better planning to approach lesions of the brainstem and may help disseminate the techniques for successful resection of intrinsic brainstem lesions.

\section{Acknowledgments}

The work described in this paper was funded by the Barrow Neurological Foundation and by the Newsome Chair in Neurosurgery Research held by Dr. Preul.

\section{References}

1. Abla AA, Lekovic GP, Turner JD, de Oliveira JG, Porter R, Spetzler RF: Advances in the treatment and outcome of brainstem cavernous malformation surgery: a single-center case series of 300 surgically treated patients. Neurosurgery 68:403-415, 2011

2. Baghai P, Vries JK, Bechtel PC: Retromastoid approach for biopsy of brain stem tumors. Neurosurgery 10:574-579, 1982

3. Bailey P, Buchanan DN, Bucy PC: Intracranial Tumors of Infancy and Childhood. Chicago: University of Chicago Press, 1939

4. Bricolo A: Brainstem tumors, in Sindou M (ed): Practical Handbook of Neurosurgery. Vienna: Springer, 2009 
5. Bricolo A, Turazzi S: Surgery for gliomas and other mass lesions of the brainstem. Adv Tech Stand Neurosurg 22:261341,1995

6. Bricolo A, Turazzi S, Cristofori L, Talacchi A: Direct surgery for brainstem tumours. Acta Neurochir Suppl (Wien) 53:148-158, 1991

7. Brown AP, Thompson BG, Spetzler RF: The two-point method: Evaluating brainstem lesions. Barrow Quarterly 12:20-24, 1996

8. Brunso-Bechtold JK, Thompson GC, Masterton RB: HRP study of the organization of auditory afferents ascending to central nucleus of inferior colliculus in cat. J Comp Neurol 197:705-722, 1981

9. Cantore G, Missori P, Santoro A: Cavernous angiomas of the brain stem. Intra-axial anatomical pitfalls and surgical strategies. Surg Neurol 52:84-94, 1999

10. Cavalcanti DD, García-González U, Agrawal A, Crawford NR, Tavares PL, Spetzler RF, et al: Quantitative anatomic study of the transciliary supraorbital approach: benefits of additional orbital osteotomy? Neurosurgery 66 (6 Suppl Operative):205-210, 2010

11. Cavalcanti DD, Kalani MY, Martirosyan NL, Eales J, Spetzler RF, Preul MC: Cerebral cavernous malformations: from genes to proteins to disease. J Neurosurg 116:122-132, 2012

12. de Oliveira JG, Lekovic GP, Safavi-Abbasi S, Reis CV, Hanel RA, Porter RW, et al: Supracerebellar infratentorial approach to cavernous malformations of the brainstem: surgical variants and clinical experience with 45 patients. Neurosurgery 66:389-399, 2010

13. Deshmukh VR, Figueiredo EG, Deshmukh P, Crawford NR, Preul MC, Spetzler RF: Quantification and comparison of telovelar and transvermian approaches to the fourth ventricle. Neurosurgery 58:ONS-202-ONS-207, 2006

14. Deshmukh VR, Rangel-Castilla L, Spetzler RF: Lateral inferior cerebellar peduncle approach to dorsolateral medullary cavernous malformation. J Neurosurg 121:723-729, 2014

15. Epstein F, McCleary EL: Intrinsic brain-stem tumors of childhood: surgical indications. J Neurosurg 64:11-15, 1986

16. Feiz-Erfan I, Horn EM, Spetzler RF: Transanterior perforating substance approach to the thalamomesencephalic junction. Neurosurgery 63:ONS69-ONS72, 2008

17. Ferroli P, Sinisi M, Franzini A, Giombini S, Solero CL, Broggi G: Brainstem cavernomas: long-term results of microsurgical resection in 52 patients. Neurosurgery 56:12031214,2005

18. Figueiredo EG, Deshmukh V, Nakaji P, Deshmukh P, Crusius MU, Crawford N, et al: An anatomical evaluation of the mini-supraorbital approach and comparison with standard craniotomies. Neurosurgery 59:ONS212-ONS220, 2006

19. Fischer B, Weber H: Express saccades and visual attention. Behav Brain Sci 16:553-567, 1993

20. Hebb MO, Spetzler RF: Lateral transpeduncular approach to intrinsic lesions of the rostral pons. Neurosurgery 66 (3 Suppl Operative):26-29, 2010

21. Hubel DH, LeVay S, Wiesel TN: Mode of termination of retinotectal fibers in macaque monkey: an autoradiographic study. Brain Res 96:25-40, 1975

22. Kumar RSV: Tuberculous brain stem abscesses in children. J Pediatr Neurol 2:101-106, 2004

23. Kyoshima K, Kobayashi S, Gibo H, Kuroyanagi T: A study of safe entry zones via the floor of the fourth ventricle for brain-stem lesions. Report of three cases. J Neurosurg 78:987-993, 1993

24. Lanzino G, Paolini S, Spetzler RF: Far-lateral approach to the craniocervical junction. Neurosurgery 57 (4 Suppl):367-371, 2005

25. Lassiter KR, Alexander E Jr, Davis CH Jr, Kelly DL Jr: Surgical treatment of brain stem gliomas. J Neurosurg 34:719725,1971

26. Matson DD, Ingraham FD Neurosurgery of Infancy and Childhood, ed 2. Springfield, IL: Thomas, 1969

27. Merchán MA, Saldaña E, Plaza I: Dorsal nucleus of the lat- eral lemniscus in the rat: concentric organization and tonotopic projection to the inferior colliculus. J Comp Neurol 342:259-278, 1994

28. Munoz DP, Wurtz RH: Fixation cells in monkey superior colliculus. I. Characteristics of cell discharge. J Neurophysiol 70:559-575, 1993

29. Mussi AC, Rhoton AL Jr: Telovelar approach to the fourth ventricle: microsurgical anatomy. J Neurosurg 92:812-823, 2000

30. Ono M, Ono M, Rhoton AL Jr, Barry M: Microsurgical anatomy of the region of the tentorial incisura. J Neurosurg 60:365-399, 1984

31. Pool JL: Gliomas in the region of the brain stem. J Neurosurg 29:164-167, 1968

32. Porter RW, Detwiler PW, Spetzler RF, Lawton MT, Baskin JJ, Derksen PT, et al: Cavernous malformations of the brainstem: experience with 100 patients. J Neurosurg 90:50-58, 1999

33. Raimondi AJ: Pediatric Neurosurgery: Theoretical Principles-Art of Surgical Techniques. New York: Springer, 1987

34. Ramina R, Mattei TA, de Aguiar PH, Meneses MS, Ferraz VR, Aires R, et al: Surgical management of brainstem cavernous malformations. Neurol Sci 32:1013-1028, 2011

35. Recalde RJ, Figueiredo EG, de Oliveira E: Microsurgical anatomy of the safe entry zones on the anterolateral brainstem related to surgical approaches to cavernous malformations. Neurosurgery 62 (3 Suppl 1):9-17, 2008

36. Sala F, Manganotti P, Tramontano V, Bricolo A, Gerosa M: Monitoring of motor pathways during brain stem surgery: what we have achieved and what we still miss? Neurophysiol Clin 37:399-406, 2007

37. Strauss C, Lütjen-Drecoll E, Fahlbusch R: Pericollicular surgical approaches to the rhomboid fossa. Part I. Anatomical basis. J Neurosurg 87:893-899, 1997

38. Wind JJ, Bakhtian KD, Sweet JA, Mehta GU, Thawani JP, Asthagiri AR, et al: Long-term outcome after resection of brainstem hemangioblastomas in von Hippel-Lindau disease. J Neurosurg 114:1312-1318, 2011

39. Yaşargil MG: Microsurgical Anatomy of the Basal Cisterns and Vessels of the Brain, Diagnostic Studies, General Operative Techniques and Pathological Considerations of the Intracranial Aneurysms. Stuttgart: Thieme, 1984

40. Zabramski JM, Kiriș T, Sankhla SK, Cabiol J, Spetzler RF: Orbitozygomatic craniotomy. Technical note. J Neurosurg 89:336-341, 1998

41. Zhou LF, Du G, Mao Y, Zhang R: Diagnosis and surgical treatment of brainstem hemangioblastomas. Surg Neurol 63:307-316, 2005

\section{Disclosure}

The authors report no conflict of interest concerning the materials or methods used in this study or the findings specified in this paper.

\section{Author Contributions}

Conception and design: Cavalcanti. Acquisition of data: Cavalcanti. Analysis and interpretation of data: Cavalcanti. Drafting the article: Cavalcanti, Kalani. Critically revising the article: Preul, Kalani, Spetzler. Reviewed submitted version of manuscript: Preul, Kalani, Spetzler. Administrative/technical/material support: Preul. Study supervision: Preul, Spetzler.

\section{Correspondence}

Daniel D. Cavalcanti, Departamento de Neurocirurgia, Instituto Estadual do Cérebro Paulo Niemeyer, Rua do Resende, 156, Rio de Janeiro, RJ, Brazil 20231-092. email: danieldc.neuro@gmail. com. 